Article

\title{
A Non-Reference Image Denoising Method for Infrared Thermal Image Based on Enhanced Dual-Tree Complex Wavelet Optimized by Fruit Fly Algorithm and Bilateral Filter
}

\author{
Yiwen Liu ${ }^{1}$, Zhongbin Wang ${ }^{1,2, *}$, Lei Si ${ }^{1, *}$ (D) , Lin Zhang ${ }^{1}{ }^{(\mathbb{D})}$, Chao $\operatorname{Tan}^{1}$ and Jing Xu ${ }^{1}$ \\ 1 School of Mechatronic Engineering, China University of Mining \& Technology, No.1 Daxue Road, \\ Xuzhou 221116, China; lyw.3400@aliyun.com (Y.L.); lin.zhang_2014@hotmail.com (L.Z.); \\ tccadcumt@126.com (C.T.); xujingcmee@cumt.edu.cn (J.X.) \\ 2 Jiangsu Key Laboratory of Mine Mechanical and Electrical Equipment, China University of \\ Mining \& Technology, No. 1 Daxue Road, Xuzhou 221116, China \\ * Correspondence: wangzbpaper@126.com (Z.W.); sileicool@163.com (L.S.); Tel.: +86-516-8359-0758 (Z.W.)
}

Received: 19 October 2017; Accepted: 13 November 2017; Published: 22 November 2017

\begin{abstract}
To eliminate the noise of infrared thermal image without reference and noise model, an improved dual-tree complex wavelet transform (DTCWT), optimized by an improved fruit-fly optimization algorithm (IFOA) and bilateral filter (BF), is proposed in this paper. Firstly, the noisy image is transformed by DTCWT, and the noise variance threshold is optimized by the IFOA, which is enhanced through a fly step range with inertia weight. Then, the denoised image will be re-processed using bilateral filter to improve the denoising performance and enhance the edge information. In the experiment, the proposed method is applied to eliminate both addictive noise and multiplicative noise, and the denoising results are compared with other representative methods, such as DTCWT, block-matching and 3D filtering (BM3D), median filter, wiener filter, wavelet decomposition filter (WDF) and bilateral filter. Moreover, the proposed method is applied as pre-processing utilization for infrared thermal images in a coal mining working face.
\end{abstract}

Keywords: non-reference image denoising; DTCWT; fruit-fly optimization algorithm; bilateral filter

\section{Introduction}

Image denoising is a very important part of image processing, and it is the basis of all subsequent processing, such as image recognition [1], object detection and tracking [2]. The denoising methods are mainly divided into spatial domain and transform domain filters. Spatial domain filters have high processing speed since they operate the pixels on the original images, such as median filter [3], wiener filter [4] and bilateral filter (BF) [5]. These algorithms can remove noise quickly, but the denoising abilities are mediocre. Transform domain filters are more robust since the images are transformed into another domain. Two major transform domain filters are Fourier transform (FT) [6] and wavelet transform (WT) [7]. WT overcomes the problem of FT that the window size is fixed, and does not change with frequency. Thus, WT gets better effect than FT. Discrete wavelet transform (DWT), proposed by Shensa [8], is widely applied in the image processing field. Dual-tree complex wavelet transform (DTCWT) proposed by N. Kingsbury in 1998 [9] can improve the DWT in several aspects. It owns three additional properties: approximate shift invariance, good directional selectivity, and limited data redundancy [10]. Recently, DTCWT has been used to eliminate the noise of images, and the method has been improved by many researchers. Shahdoosti et al. [11] proposed a denoising method using the dual contourlet transform (DCT), which combined the directional filter bank (DFB) and DTCWT. The bivariate shrinkage function (BSF) with local variance estimation was adopted 
in [12] to process wavelet coefficients of DTCWT and the denoised images were reconstructed by the shrunk wavelet coefficients. Through changing the window size and shape of DTCWT, Liu et al. [13] acquired good quality of denoised images. However, these methods cannot reach the best denoising performance since the parameters are fixed. Therefore, intelligent methods that search the best parameters through the optimization algorithm have been proposed by some researchers.

In recent years, optimization problems are more and more extensive and many meta-heuristic algorithms has been proposed, such as genetic algorithm [14], ant colony algorithm [15], fish swarm algorithm [16], particle swarm algorithm [17], Virus colony search (VCS) algorithm [18], fire-fly algorithm [19], and so on. Among these algorithms, fruit-fly optimization algorithm (FOA), proposed by Professor Pan Wenchao in 2011 [20], has the advantages of simple structure, easy parameter setting, and fast convergence rate. Thus, FOA is widely used in economic management [21], forecasting [22], planning [23], etc. Nevertheless, similar to other optimal evolutionary algorithms, the original FOA may sink into local optima due to fixed fly step size. Meanwhile, the fitness function is a key problem that affects the denoising performance. Some researchers have put forward some examples. However, Most of them are full-reference. In practice, reference images cannot be acquired and these methods are not capable for practical usage.

Moreover, the original thermal images cannot be used for analyzing because of the low sharpness, noise problem and inhomogeneous. Therefore, it is a significant task to create a method for thermal images that is capable of acquiring best image denoised performance without reference images. In this work, a non-reference image denoising method based on enhanced dual-tree complex wavelet optimized by fruit fly algorithm and bilateral filter (IFOA-DTCWT-BF) is proposed and the method is compared with DTCWT and other denoising algorithms. Some common objective indexes are used to analyze the performance of these algorithms.

The rest of this paper is organized as follows. In Section 2, some related works are outlined based on the literature. In Section 3, the basic theories of DTCWT, FOA and bilateral filter are presented. In Section 4, the image denoising method based on IFOA-DTCWT-BF is elaborated, and a fly step range improved by inertia weight is performed. In Section 5, experiments are conducted and the proposed denoising method is tested, and an application is performed. Some conclusions and outlooks are summarized in Section 6.

\section{Literature Review}

This paper is mainly concerned with three research streams: image denoising method, dual-tree wavelet transform, and fruit fly optimization algorithm. In this section, we will summarize the relevant literature.

\subsection{Image Denoising Method}

In recent years, image denoising becomes an urgent need in image processing field. Many creative methods are put forward to wipe out the image noise. HR. Shahdoosti and SM. Hazavei [11] applied the hidden Markov tree (HMT) with mixtures of one-sided exponential densities to denoise images. To excavate the spatial information in hyperspectral image, a novel denoising method which integrates superpixel segmentation (SS) into low-rank representation (LRR), is presented in [24]. Because of the advantage in preserving texture in image, total variation is widely applied in the problems of image denoising [25]. Jia Zhi-Gang et al. [26] proposed a new TV-Stokes model for image deblurring with a good geometry explanation. In reality, the noise models of noisy images are unknown beforehand. Zhu et al. [27] consider the problem and proposes a novel blind image denoising algorithm to recover the clean image from noisy one with the unknown noise model. Kernel greedy algorithm for robust denoising (KGARD), put forward in [28], employs sparse modeling arguments to explicitly model and estimate the outliers, adopting a greedy approach. Recently, intelligent algorithms are applied in the image denoising field. Zhang Lin et al. [29] proposed a non-model dual-tree wavelet thresholding 
for image denoising that uses the improved chaotic drosophila optimization algorithm (CDOA) to estimate the noise variance.

\subsection{Dual-Tree Complex Wavelet Transform}

DTCWT is suitable for image processing applications [9]. In [30], DTCWT is used to create an effective contrast enhancement method and achieves a good performance in low illumination image enhancement. A new method for the interpolation of a full high-definition (HD) image which applied DTCWT to decompose the low-resolution image into different sub-bands was presented in [31]. To acquire good performance, the Wiener filter is used to remove the noise component of the dual-tree complex wavelet transform (DTCWT) coefficients [32]. In [33], the DTCWT coefficients are preprocessed by median filtering. Zhao et al. [34] presented a novel biometric image encryption algorithm based on DTCWT and compressed sensing. To produce better fusion effects, Q-shift DTCWT was proposed to simplify the construction of filters in DTCWT [35].

\subsection{Fruit Fly Optimization Algorithm}

FOA is easy to understand and has lower computational complexity. That is why FOA can be successfully applied in many areas such as the financial distress model solving [20], annual power load forecasting [36], the multidimensional knapsack problem solving [22], tuning of PID controller [37], neural network training [38] and so on. In [39], W Lijuan et al. proposed a novel approach, namely SFOASVR, which hybridizes SVR model with FOA and the seasonal index adjustment to forecast monthly tourist flow. Niu jinwei et al. [40] applied FOA to optimize the operation of the Texaco gasification process. However, the original FOA also has the possibility of falling into local extreme due to the fixed fly range and limited search space. To cover these problems, many improved methods have been proposed. Niu et al. [41] divided fruit group of FOA into two parts and used Cauchy mutation process to make fruit fly individuals variant. Xu et al. [42] improved the fly distance range $(F R)$ of the FOA by using normal distribution $F R \sim \mathrm{N}\left(0, L^{2}\right)$. A novel multi scale cooperative mutation FOA was proposed by Zhang et al. [43] to tackle the limitation of local optimum. In [22], the parallel search is employed to balance exploitation and exploration of FOA. Wang Lin et al. [44] applied a level probability policy and a new mutation parameter to balance the population diversity and stability of FOA.

\subsection{Discussion}

Although the above research works are valuable, there are still some drawbacks remained. First, the denoising methods were improved by optimization algorithms and acquired better performances in recent decades, at the same time they were dragged down by the great amount of calculation and slow convergence rate of the optimization algorithms. Second, improved denoising algorithms always need reference images to get good performances, while, in practice, reference images do not exist. Third, despite the fine iteration rate and easy encoding, the original FOA is still likely to fall into local extreme due to the settled parameters. Moreover, the images processed by DTCWT always have small speckles which influence the quality of denoised images.

Therefore, an adaptive dual-tree complex wavelet threshold denoising method for non-reference image denoising based on improved fruit fly algorithm and bilateral filter is proposed in this paper, and a series of simulations and applications prove the effectiveness of the method.

\section{Basic Theory}

\subsection{Dual-Tree Complex Wavelet Transform Based on Bivariate Shrinkage Function}

When processing image with DTCWT, the image is resolved into a desired level by two separable 2D DWT branches, branch $a$ (real part) and branch $b$ (imagery part), whose filters are explicitly designed to meet the Hilbert pair requirement. 
As shown in Figure 1, the $h_{0}(n), h_{1}(n)$ are the low pass filter and high pass filter of the filter bank above which produce the scale coefficient and wavelet coefficient of the real part, while the $g_{0}(n), g_{1}(n)$ are the low pass filter and high pass filter of the filter bank below that create the scale coefficient and wavelet coefficient of the imaginary part. In the first stage, the image has a line transformation through filter $\left\{g_{0}(n), g_{1}(n)\right\}$, then it has a rank transformation through filter $\left\{\mathrm{h}_{0}(\mathrm{n}), \mathrm{h}_{1}(\mathrm{n})\right\}$. In the second stage, the image has a line transformation through filter $\left\{h_{0}(n), h_{1}(n)\right\}$, then has a rank transformation through filter $\left\{g_{0}(n), g_{1}(n)\right\}$. The decomposition at each stage produces one low-pass sub band LL and three high-pass sub bands HL, LH, and HH. The sum or difference of each pair of sub bands forms low-frequency wavelet coefficients and six wavelet transform coefficients in six directions. Then, bivariate shrinkage function is applied to process the wavelet coefficients.

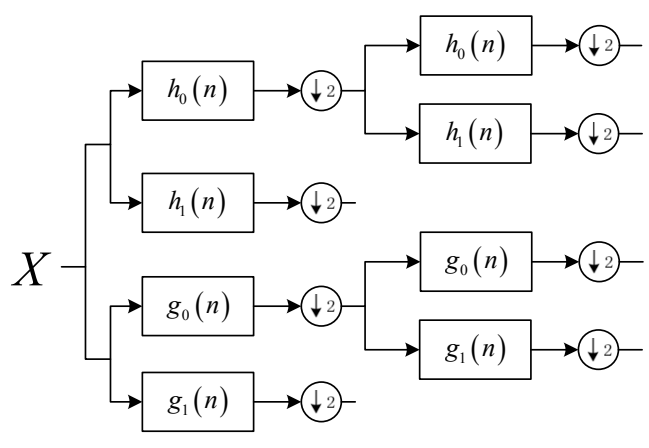

(A)

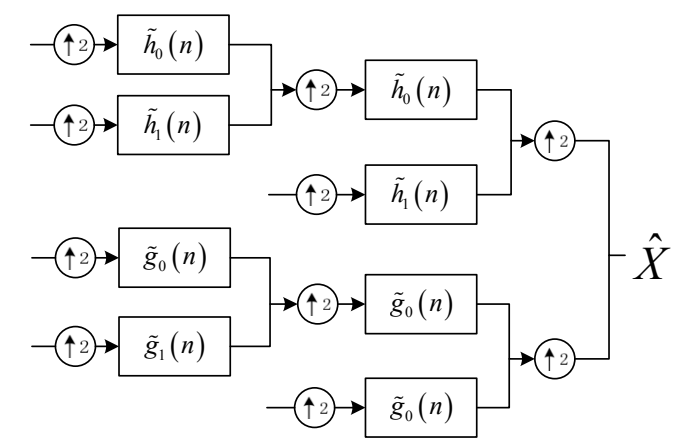

(B)

Figure 1. Dual-tree complex wavelet transform: (A) analysis filter bank; and (B) synthesis filter bank.

Bivariate shrinkage function was proposed by Sendur L and Selesnick I W [45] in 2002. The DTCWT with bivariate shrinkage function can be described as follows.

Assuming that the image is contaminated by noise, the noised image can be denoted as:

$$
f=x \circ n,
$$

where the $f$ is a noisy image; $\circ$ is the operator represents corresponding to different noise models, e.g., Additive noise is + , and multiplicative noise is $\times ; x$ is the original image; and $n$ is the noise. It is transformed into dual-tree complex wavelet domain:

$$
y=\omega+n,
$$

where $y$ stands for the wavelet coefficient of the observed image, $\omega$ represents the wavelet coefficient of the original image (to be estimated), and $n$ is the noise wavelet coefficient which is independent and identically distributed and obeys the $N\left(0, \sigma^{2}\right)$. factor $y$ :

Using the maximum a posteriori probability estimate (MAP), the $\omega$ is estimated from the noise

$$
\hat{\omega}(y)=\arg \max _{\omega} p_{\omega \mid y}(\omega \mid y),
$$

where $p_{\omega \mid y}(\omega \mid y)$ is the posterior probability function.

According to Bayesian estimation theory, Formula (3) can be written:

$$
\begin{aligned}
& \hat{\omega}(y)=\arg \max _{\omega}\left[p_{n}(n) \cdot p_{\omega}(\omega)\right], \\
& p_{n}(n)=\frac{1}{2 \pi \sigma^{2}} \exp \left(-\frac{n_{1}^{2}+n_{2}^{2}}{2 \sigma^{2}}\right),
\end{aligned}
$$


$p_{\omega}(\omega)$ is the bivariate probability distribution function that proposed in [45]:

$$
p_{\omega}(\omega)=\frac{3}{2 \pi \sigma_{\omega}^{2}} \cdot \exp \left(-\frac{\sqrt{3}}{\sigma} \sqrt{\omega_{1}^{2}+\omega_{2}^{2}}\right),
$$

$\omega_{1}$ is the wavelet coefficient, $\omega_{2}$ is the coefficient of the parent node at the next level of $\omega_{1}$, and $\sigma_{\omega}^{2}$ is the variance of the signal and is related to the location of the wavelet coefficient. The maximum posterior probability estimate of $\omega_{1}$ can be obtained:

$$
\begin{gathered}
\hat{\omega}_{1}=\frac{\left(\sqrt{y_{1}^{2}+y_{2}^{2}}-\frac{\sqrt{3} \sigma^{2}}{\sigma_{\omega}}\right)_{+}}{\sqrt{y_{1}^{2}+y_{2}^{2}}} \cdot y_{1}, \\
(g)_{+}=\left\{\begin{array}{l}
0, g<0 \\
g, \text { otherwise }
\end{array}\right.
\end{gathered}
$$

This is the bivariate shrinkage function.

The rectangular window region $N(k)$ centered on the first $k$ wavelet coefficients is defined. The signal variance $\sigma_{\omega}^{2}$ of this region is estimated by the wavelet coefficients in the $N(k)$ region, and the noise variance $\sigma^{2}$ is estimated by the median filtering method:

$$
\hat{\sigma^{2}}=\frac{\operatorname{Median}\left(\left|y_{i}\right|\right)}{0.6745}, y_{i} \in \text { subband } H H,
$$

where subband $H H$ is an aggregate of wavelet coefficients in the $N(k)$ region.

From the observation model, we can obtain: $\sigma_{y}^{2}=\sigma_{\omega}^{2}+\sigma^{2}$, the empirical estimation of $\sigma_{y}^{2}$ is:

$$
\hat{\sigma}_{y}^{2}=\frac{1}{M} \sum_{y_{i} \in N(k)} y_{i}^{2}
$$

$M$ is the size of the rectangle region $N(k)$, and the $\sigma_{\omega}$ is estimated to be:

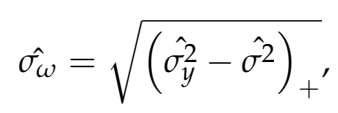

After the noisy image processed by DTCWT, the wavelet coefficients of DTCWT is handled with the local adaptive bivariate shrinkage model which has local variance estimation, and then the denoised image can be obtained via inverse DTCWT of new wavelet coefficients.

\subsection{Fruit Fly Optimization Algorithm}

Fruit fly Optimization Algorithm (FOA), similar to other biomimetic interactive evolutionary computation algorithm, simulates the foraging process of fruit fly. The procedure of basic FOA can be summarized as follows and the schematic diagram is showed in Figure 2.

Step 1.1: Initialize the parameters: population size $P S$, maximum iteration number $I N_{\max }$, location range $L R$ and the fly distance range $F R$.

The initial position of the fruit fly group is represented as follows:

$$
\left\{\begin{array}{l}
X \_a x i s=\operatorname{rand}(L R) \\
Y \_a x i s=\operatorname{rand}(L R)
\end{array},\right.
$$


Step 1.2: An individual fruit fly uses its olfactory sensation to find food, the random searching direction and distance is given as follows:

$$
\left\{\begin{array}{l}
X_{i}=X_{-} \text {axis }+\operatorname{rand}(F R) \\
Y_{i}=Y_{-} a x i s+\operatorname{rand}(F R)
\end{array}\right.
$$

Step 1.3: Because of the unknown location of the food, the distance to the origin of coordinates $\left(\right.$ Dist $\left._{i}\right)$ and the judgment value of smell concentration $\left(S_{i}\right)$ are calculated as follows:

$$
\text { Dist }_{i}=\sqrt{X_{i}^{2}+Y_{i}^{2}}, S_{i}=1 / \text { Dist }_{i}
$$

Step 1.4: The fruit fly with maximum smell concentration among the fruit fly group searches food according to the smell concentration judgment function (or called fitness function), which represents as follows:

$$
\operatorname{Smell}(i)=\operatorname{function}\left(S_{i}\right),[\text { bestsmell, bestindex }]=\max (\operatorname{Smell}(i)),
$$

where bestsmell donates the maximal smell concentration, bestindex is the corresponding fruit fly number and Smell is the smell concentration of the group.

Step 1.5: The new smell concentration is compared with the previous best smell concentration; if it is better than the last generation, the current best location and smell concentration can be presented as follows:

$$
\text { smellbest }=\text { bestsmell, }\left\{\begin{array}{l}
X_{-} \text {axis }=X(\text { bestindex }) \\
Y \_a x i s=Y(\text { bestindex })
\end{array},\right.
$$

Otherwise, repeated Step 1.2 to Step 1.4 to find other better smell concentrations.

Step 1.6: When the smell concentration reaches the preset precision value or the iteration number reaches the maximal IN, the circulation stops. Otherwise, repeat the process from Step 1.2 to Step 1.5.

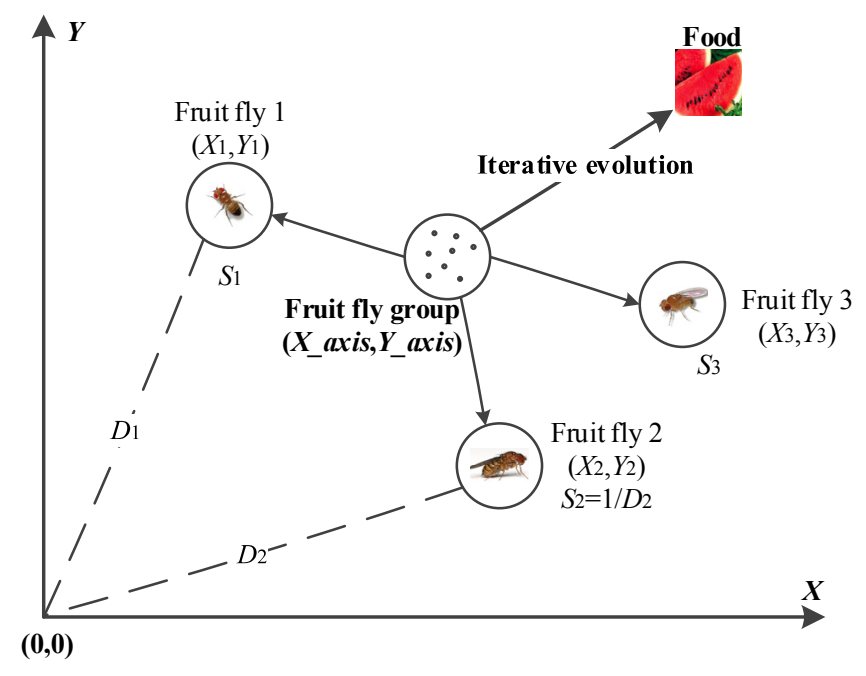

Figure 2. Fruit fly optimization algorithm.

\subsection{Bilateral Filter Algorithm}

As a kind of nonlinear spatial filter, $\mathrm{BF}$ is able to achieve uniform spatial filtering and reserve the edge information of an image. BF considers both the grey value of the point in the neighborhood scope, and the distance from center of geometric. Thus, the formula of grey value after filtering is presented as follows:

$$
h_{x}(x)=k^{-1} \iint f(\xi) c(\xi-x) s(f(\xi)-f(x)) d \xi,
$$


where $k$ is the normalization coefficient, the expression is as follows:

$$
k(x)=\iint c(\xi-x) s(f(\xi)-f(x)) d \xi
$$

in which $h_{x}(\cdot)$ is the corresponding point of the grey value before filtering, and $x$ is the value after filtering. $c(\cdot)$ is the space similarity value of the center point with its neighborhood points, $s(\cdot)$ is the gray similarity value of the center point with its neighborhood points.

The functions $c(\cdot)$ and $s(\cdot)$ can be implemented using Gaussian function, which can be defined as follows:

$$
\begin{gathered}
c(\xi-x)=e^{-\frac{1}{2}\left(\frac{\|\tilde{\xi}-x\|}{\sigma}\right)^{2},} \\
s(f(\xi)-f(x))=e^{-\frac{1}{2}\left(\frac{\|f(\tilde{\xi})-f(x)\|}{\sigma}\right)^{2},}
\end{gathered}
$$

\section{The Proposed Method}

In this section, the architecture of proposed method is presented and the flow of the proposed method is described as well. To enhance the threshold optimization ability of proposed method, an improved FOA is proposed.

\subsection{Architecture of the Proposed Method}

This algorithm is a new method that deals with noisy images without reference images. The architecture of algorithm can be divided into two parts. In the first part, DTCWT enhanced by IFOA (IFOA-DTCWT) is applied to remove noise. Since there are no reference images, the denoised images can only compare with the noisy images to estimate the denoising performance. The IFOA searches the noise variance randomly, and a new wavelet coefficient is gained through the bivariate shrinkage function with the noise variance. Then, the denoised image is obtained through the inverse DTCWT of the new wavelet coefficient. The process continues until the optimized denoised image is acquired. After being denoised by IFOA-DTCWT, many processing areas of the square shape are remained in the denoised image. Then in the second part, bilateral filter is applied to smooth the area and enhance the edge information of the image at the same time. The combination of the two methods acquired good performance. The architecture is shown in Figure 3.

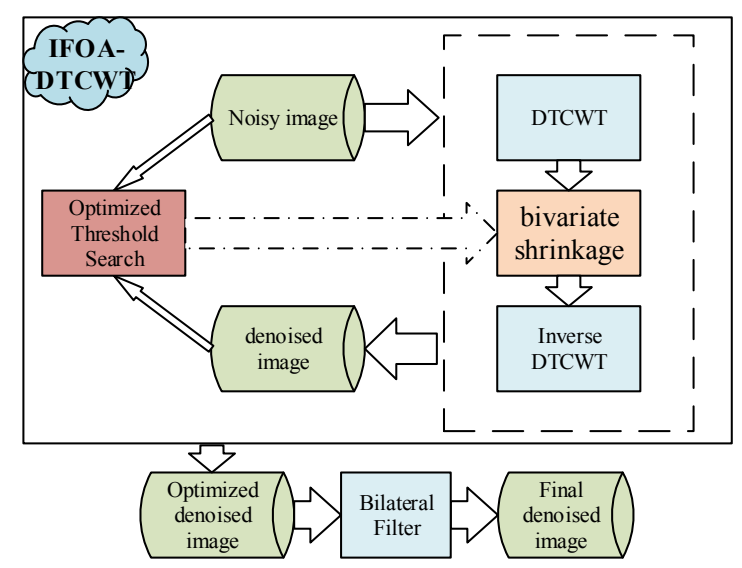

Figure 3. Architecture of enhanced dual-tree complex wavelet optimized by fruit fly algorithm and bilateral filter (IFOA-DTCWT-BF).

\subsection{Improved $F O A$}

The fly step range affects convergence speed and search ability of fruit fly optimization algorithm. The global search ability of the algorithm will be obvious improved when fly step range is large enough. 
The problem is that its speed will slow down. In the condition of small fly step range, the algorithm convergences quickly but it will be easy to fall into local optimum.

In this article, an inertia weight is applied for modification of fly step range to balance the global search ability and search speed of FOA. While in initial stage of the iteration, large inertia weight is applied to make FOA have large step range. In the late stage of the iteration, the inertia weight diminishes and makes the step range small. In this way, FOA is intended to have better global search ability and be more precise in local development. Some inertia weight functions are presented as follows:

$$
\begin{gathered}
\omega(\mathrm{g})=1-(1 \times(g-1)) / g_{\max } \\
\omega(\mathrm{g})=1-\left((g-1) / g_{\max }\right)^{2}, \\
\omega(\mathrm{g})=\cos \left(\frac{\pi}{2}(g-1) / g_{\max }\right),
\end{gathered}
$$

where $g$ is the current number of iteration, and $g_{\text {max }}$ is the maximum iteration number. The three inertia weights above are applied to improve FOA and formed IFOA1, IFOA2 and IFOA3. The flowchart of improved FOA is presented in Figure 4.

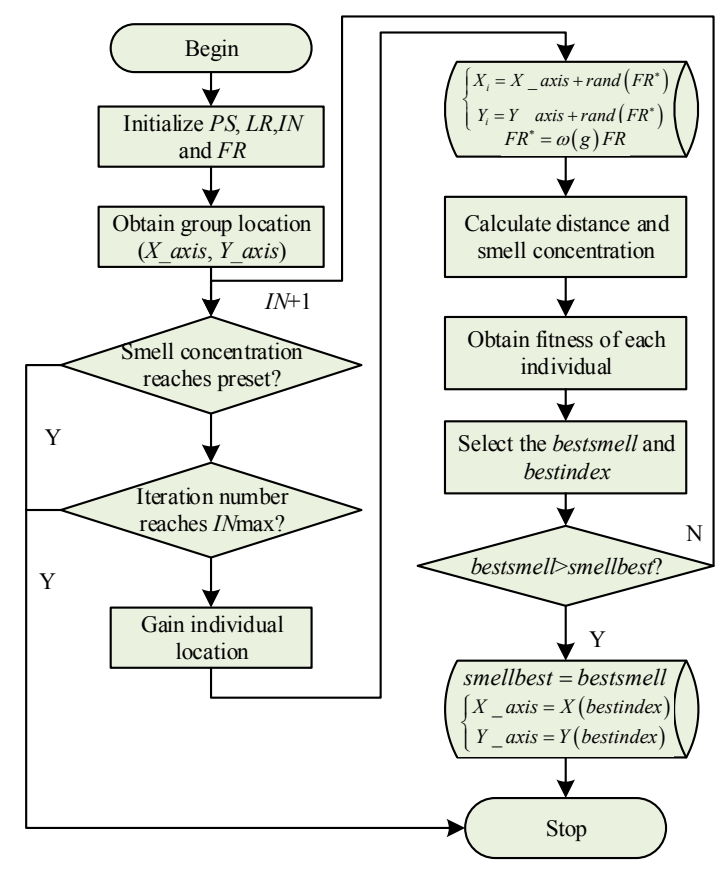

Figure 4. Flowchart of the improved fruit fly optimization algorithm.

\subsection{Flow of the Proposed Method}

The proposed IFOA-DTCWT-BF can be summarized as follows:

Step 2.1: The noisy image is first transformed into a gray image. The parameters of IFOA are initialized, like population size $P S$, maximum iteration number $I N_{\max }$, location range $L R$ and the fly step range $F R$.

Step 2.2: The location of each fly individual is gained through the fly group and $F R$. The distance and smell concentration of each fly individual are calculated according to Equation (14). Each smell concentration is considered as a potential threshold. A new threshold is just a new noise variance. Then, the noisy image is denoised through DTCWT and bivariate shrinkage function of the new variance. To evaluate the performance, the fitness function $f$ is calculated as follows:

$$
f=1 / 10 \log \frac{255^{2}}{M S E} d B
$$


where MSE is the mean square error between the noisy image and denoised image with size $m \times n$, and it can be calculated as follows:

$$
M S E=\frac{1}{m \times n} \sum_{x=1}^{m} \sum_{y=1}^{n}[f(x, y)-\hat{f}(x, y)]
$$

where $f(x, y)$ is the noisy image before processing, $\hat{f}(x, y)$ is the denoised image using proposed IFOA-DTCWT. The fitness function $f$ reaches the maximal when MSE is the maximum.

Step 2.3: Fruit fly with maximal fitness is selected as bestsmell. If the present bestsmell is bigger than the former value, smellbest, the corresponding coordinates are updated. Otherwise, smellbest, $X_{-a x i s}$ and $Y_{\_}$axis are reserved.

Step 2.4: If the ending conditions are researched, smellbest, $X_{\_}$axis and $Y_{\_}$axis are treated as the optimum. Otherwise, Steps 2.2 and 2.3 are repeated.

Step 2.5: The optimal threshold is regarded as the noise variance of noisy image and the noisy image is denoised by the DTCWT based on bivariate shrinkage function.

Step 2.6: After being denoised by the DTCWT, the image will be processed with bilateral filter. Then the final denoised image is obtained.

The Pseudocode code of the proposed method is demonstrated as Algorithm 1:

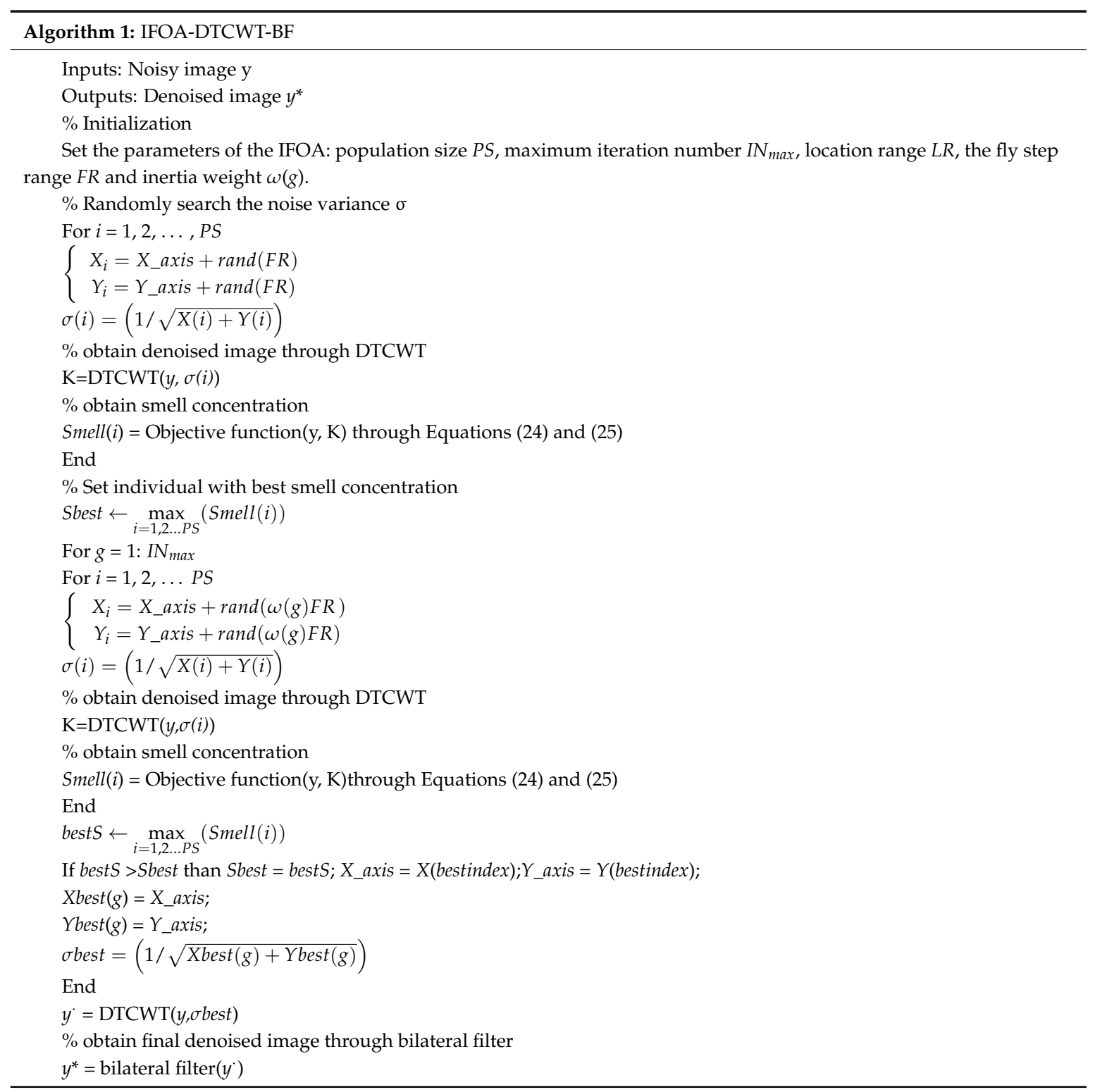




\section{Experiment and Application}

In this part, the improved FOA was tested and the proposed method IFOA-DTCWT-BF was used to remove noise of the images which were polluted by Gaussian noise and Speckle noise. Moreover, the denoising method was applied to denoise the real images from the surface experiment. To test the performance of improved fruit fly algorithm, a Schaffer function that has only one minimum point $\min f(0,0)=1$ was used to test the search ability of algorithms. The Schaffer function is always used to test the algorithms' performance, since there are many local extremum points around the minimum point, forming two loop ditches. The optimization algorithms are easy to fall into the local minimum point. In addition, the typical image denoising methods, e.g., DTCWT, block-matching and 3D filtering (BM3D), median filtering, wiener filtering, wavelet decomposition filtering (DWF) and bilateral filtering (BF), were applied to compare with the proposed method. Experiments and algorithm implementation were performed using Matlab, the experiment configuration was showed in Table 1, and the testing gray images were showed in Figure 5.

Table 1. Experiment configurations. RAM: random-access memory.

\begin{tabular}{cc}
\hline Index & Configuration \\
\hline Processor & Intel Xeon E5506, $2.13 \mathrm{GHz}$ \\
RAM & $8 \mathrm{G}$ \\
System & Win7, $\times 64$ \\
Software & Matlab \\
\hline
\end{tabular}

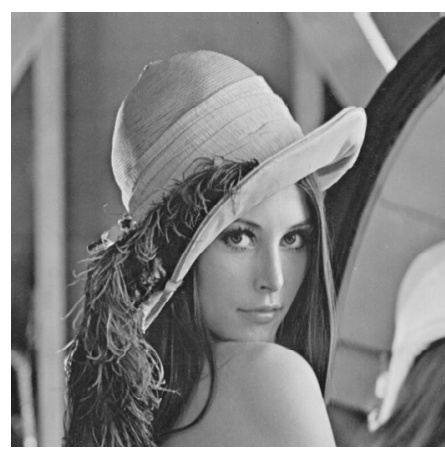

(A) Lena

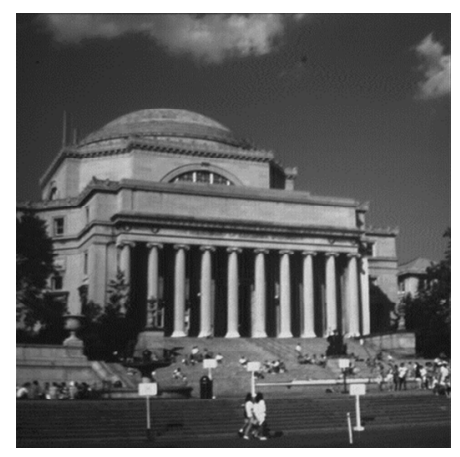

(B) Columbia

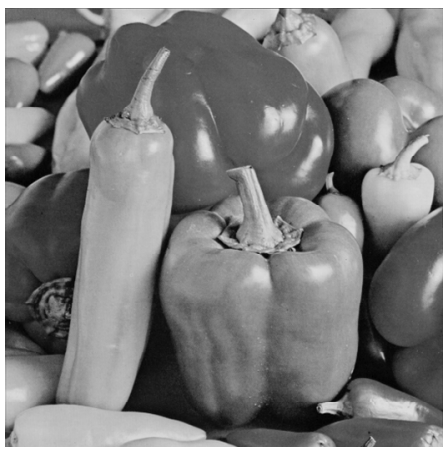

(C) Peppers

Figure 5. Original testing gray images (size: $512 \times 512$ ). (A) Lena; (B) Columbia; (C) Peppers.

Some key parameters of IFOA are initialized: maximum number of generation is 30, population size is 20 , location range is $[-10,10]$, etc.

\subsection{Algorithm Performance Testing}

To compare the improved FOA with previous FOA and test the performances of three different inertia weights, the four algorithms were tested by searching the minimum points of Schaffer function to compare their performances. The Schaffer function has only one minimum point $\min (0,0)=-1$, and many local extremum points around the minimum point form two loop ditches. The function values are 0.990283 and 0.962776 respectively, so it is easy to fall into these tiny points.

Some parameters of FOA: maximum number of generation was 90, population size was 20, the initial location range was $[-1.0,1.0]$, fly step range was $[-2.0,2.0]$. The three IFOAs changed the original fly step range with different inertia weights. Because of the inertia weight, the fly step range of improved FOA could be set two times larger than normal step or more. The improved fly range can be expressed as $\omega(\mathrm{g})[-5.0,5.0]$. In Figure 6, IFOA had faster speed in the early stage of the global search. 
Then, in the later local optimization, IFOA had higher precision of search capabilities and found the extremum more accurately.

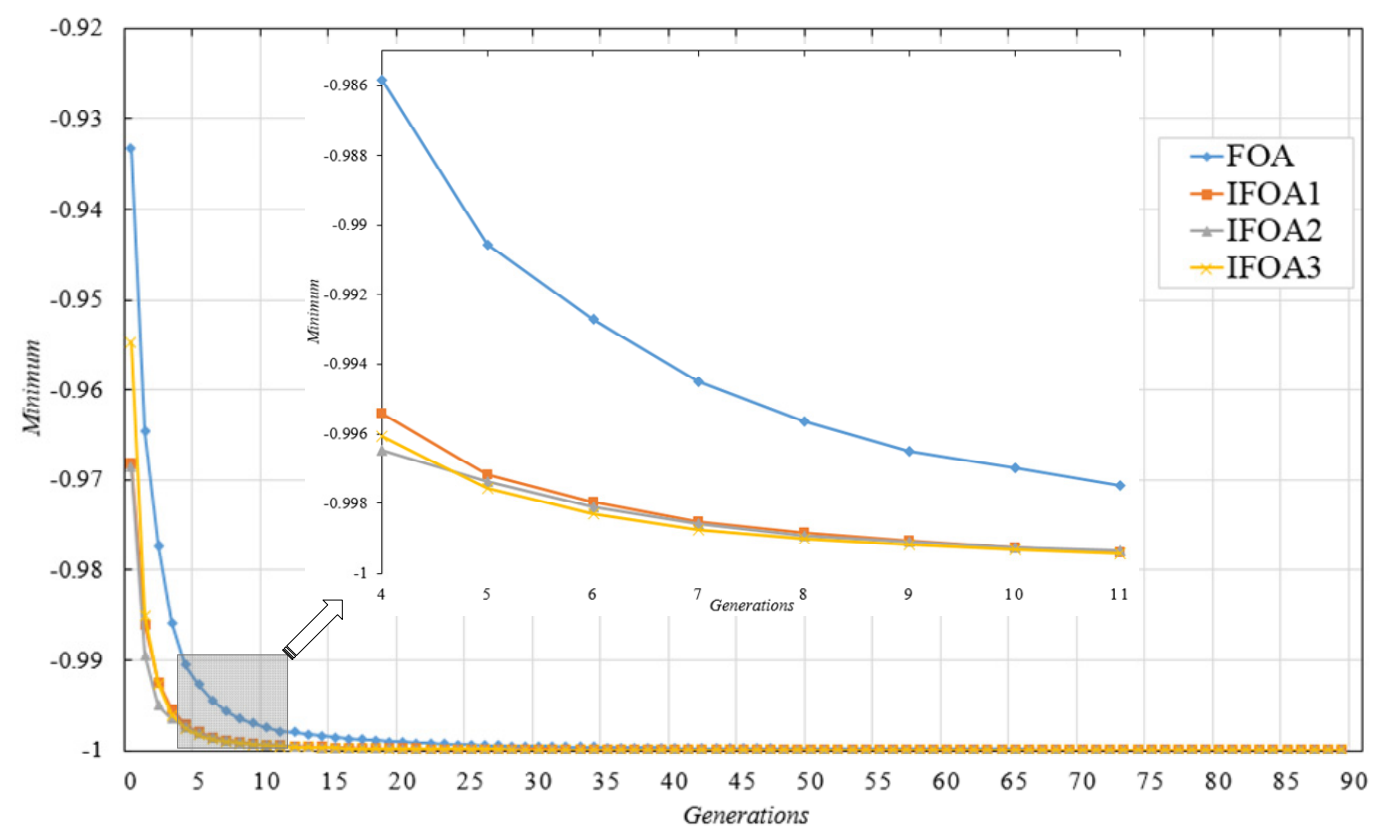

Figure 6. Optimization result of FOA and IFOAs with different inertia weights.

Figure 6 demonstrates that improved FOAs were faster than original FOA in search for extremum at the beginning. When in the later of the process, the local search became more detailed to find accurate extremum. Moreover, different inertia weights had different performances. The IFOA1 and IFOA2 searched quickly, and the IFOA3 was getting faster and faster and surpassed the two algorithms at the fifth generation. In Figures 7-10, the search route of IFOA was much wider than original FOA. The IFOA2 and IFOA3 had almost same search route. Overall, the fly step improved by inertia weights had good performance.

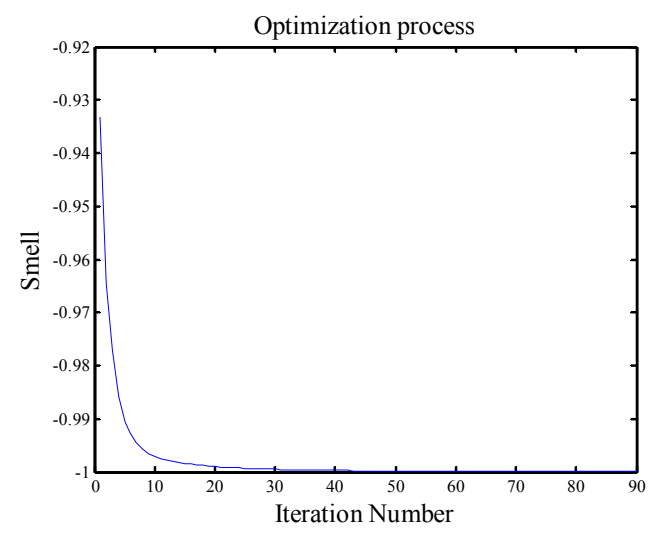

(A)



(B)

Figure 7. (A) Optimization process of FOA; and (B) fruit fly flying route of FOA. 


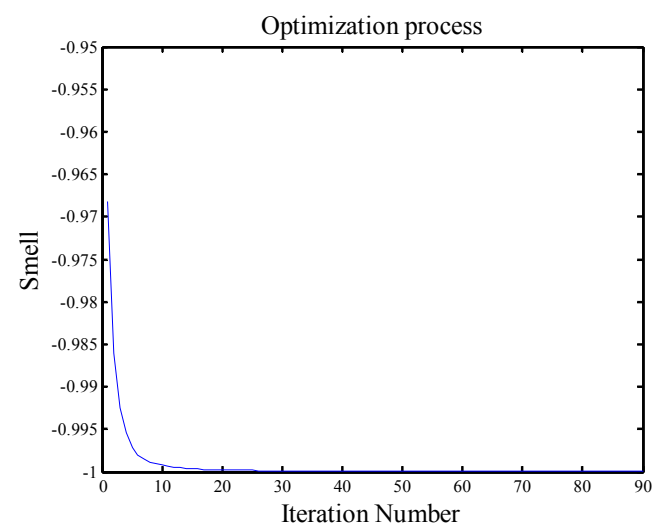

(A)

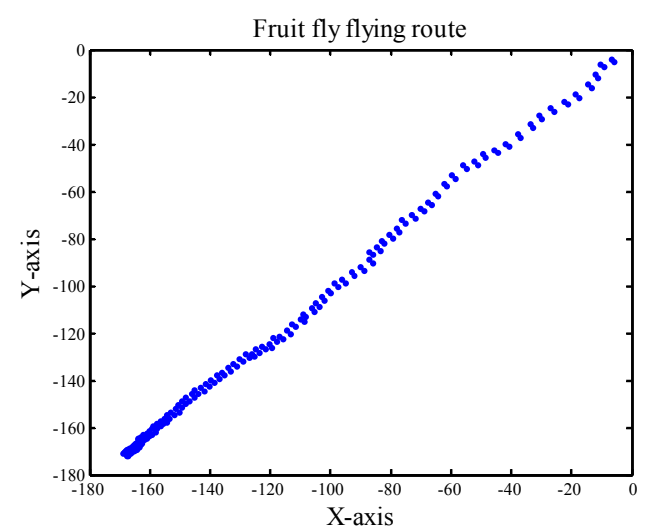

(B)

Figure 8. (A) Optimization process of FOA1; and (B) fruit fly flying route of FOA1.

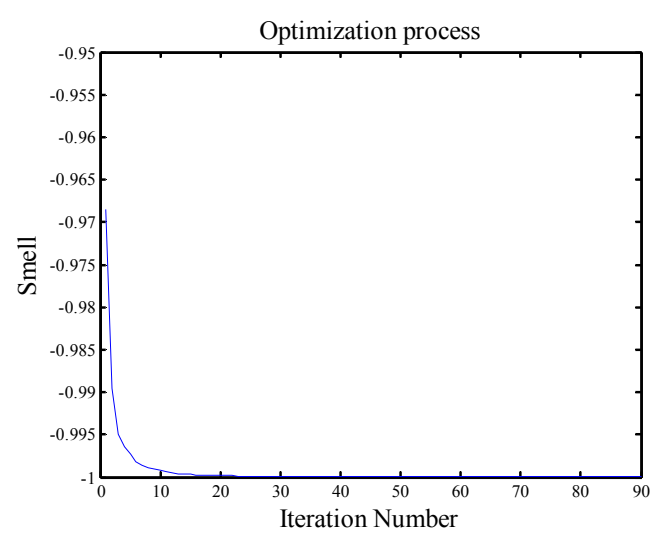

(A)

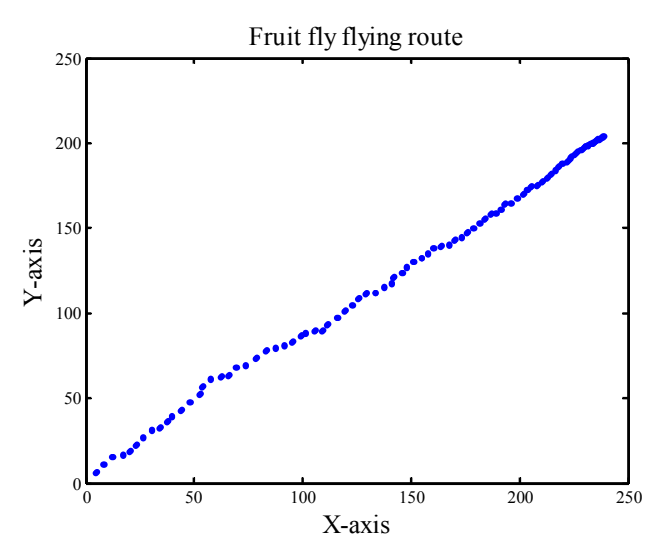

(B)

Figure 9. (A) Optimization process of FOA2; and (B) fruit fly flying route of FOA2.

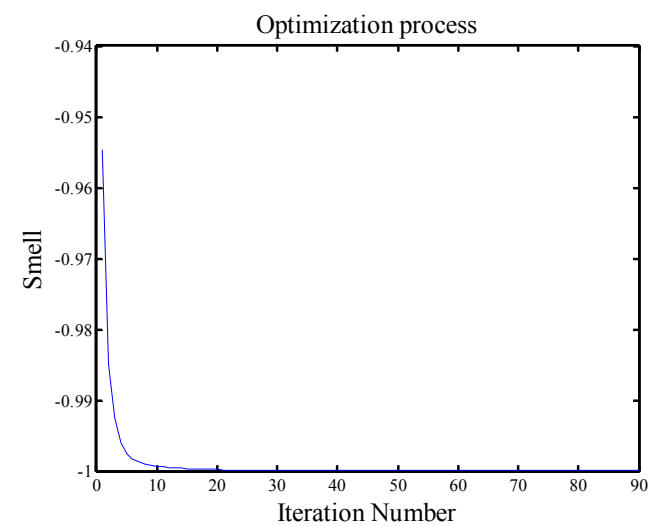

(A)

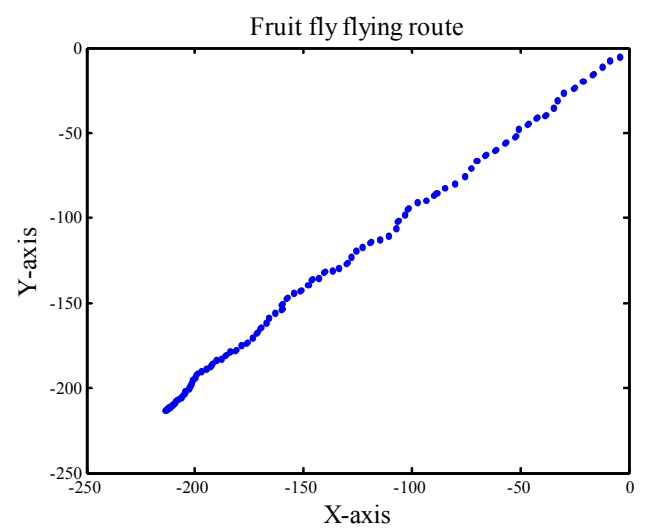

(B)

Figure 10. (A) Optimization process of FOA3; and (B) fruit fly flying route of FOA3.

Then, the FOA and IFOAs were applied in denoising algorithm. Firstly adding Gaussian noise on the original image formed the noisy image. It could be described as $g(x, y)=f(x, y)+v(x, y)$. To simulate the Gaussian noise on the original image, the test image Lena.bmp (size: $512 \times 512$ ) was polluted with zero-mean Gaussian noise with the noise variance $\sigma=0.1$. To compare the optimization ability of the FOA and IFOAs, four algorithms were applied to optimize the threshold of DTCWT to remove Gaussian noise on Lena image. The window size of the DTCWT was $5 \times 5$ which was 
convenient for bilateral filter to remove the small noise in the small areas. Some parameters of FOA include: maximum number of generation was 30 , population size was 20 , the flies' initial location range was $[-10.0,10.0]$, and fly step range of $[-1.0,1.0]$. IFOA parameters include: the fly step length of $\omega(g)[-2.0,2.0]$, and other parameters were same as FOA.

In Figure 11, the IFOAs convergences faster than FOA, and searched more meticulous than FOA. It showed that IFOA possessed the ability of fast global search and precise local search. The IFOA2 with the inertia weight of Formula (22) had the best performance in searching the optimal threshold of the denoising method. As a result, we used IFOA2 to improve the proposed method.

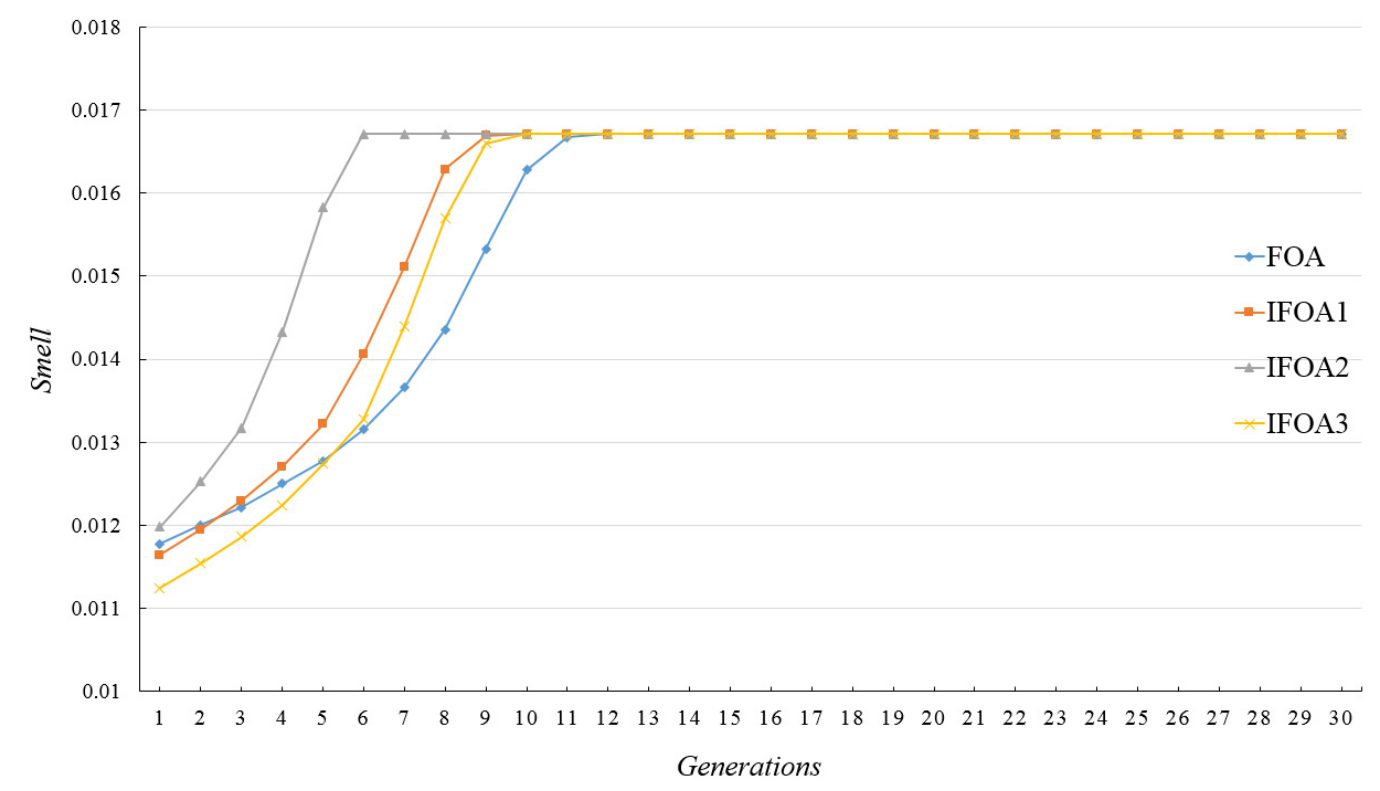

Figure 11. Optimization result using FOA and IFOAs to remove Gaussian noise from noisy image (Lena).

\subsection{Experiment with Gaussian Noise}

To compare denoising performance, Gaussian noise with different noise variance was added to different original images, and other denoising methods such as DTCWT, median filter, wiener filter, bilateral filter, and wavelet decomposition filter were used to remove the noise of the image. The image quality evaluation strategies were peak signal to noise ratio (PSNR) and two-dimensional correlation coefficient of image matrix $r$. The PSNR and $r$ here were calculated from denoised images and original images. Denoising results are listed in Tables $2-4$.

Table 2. Comparison of the five methods (Lena with Gaussian noise). WDF: wavelet decomposition filter; BM3D: block-matching and 3D filtering; DTCWT: dual-tree complex wavelet transform; IFOA-DTCWT-BF: enhanced dual-tree complex wavelet optimized by fruit fly algorithm and bilateral filter.

\begin{tabular}{|c|c|c|c|c|c|c|c|c|}
\hline \multirow[b]{2}{*}{ Methods } & \multicolumn{2}{|c|}{$\sigma=0.01$} & \multicolumn{2}{|c|}{$\sigma=0.05$} & \multicolumn{2}{|c|}{$\sigma=0.1$} & \multicolumn{2}{|c|}{$\sigma=0.2$} \\
\hline & PSNR & $\mathbf{r}$ & PSNR & $\mathbf{r}$ & PSNR & $\mathbf{r}$ & PSNR & $\mathbf{r}$ \\
\hline Noised & 20.07 & 0.8837 & 13.64 & 0.6441 & 11.32 & 0.5053 & 9.518 & 0.3731 \\
\hline Median & 27.86 & 0.9766 & 23.41 & 0.9362 & 20.93 & 0.8945 & 18.40 & 0.8281 \\
\hline Wiener & 27.70 & 0.9726 & 20.90 & 0.8881 & 18.66 & 0.8105 & 16.93 & 0.7062 \\
\hline WDF & 27.48 & 0.9745 & 23.94 & 0.9411 & 21.77 & 0.9008 & 19.57 & 0.8289 \\
\hline Bilateral & 25.44 & 0.9600 & 14.93 & 0.6971 & 11.98 & 0.5345 & 9.863 & 0.3877 \\
\hline BM3D & 31.96 & 0.9909 & 16.02 & 0.7419 & 12.52 & 0.5543 & 10.26 & 0.3975 \\
\hline DTCWT & 31.01 & 0.9887 & 26.51 & 0.9687 & 24.30 & 0.9525 & 21.70 & 0.9229 \\
\hline IFOA-DTCWT-BF & 28.66 & 0.9807 & 26.34 & 0.9685 & 24.15 & 0.9512 & 21.57 & 0.9155 \\
\hline
\end{tabular}


Table 3. Comparison of the five methods (Columbia with Gaussian noise).

\begin{tabular}{|c|c|c|c|c|c|c|c|c|}
\hline \multirow[b]{2}{*}{ Methods } & \multicolumn{2}{|c|}{$\sigma=0.01$} & \multicolumn{2}{|c|}{$\sigma=0.05$} & \multicolumn{2}{|c|}{$\sigma=0.1$} & \multicolumn{2}{|c|}{$\sigma=0.2$} \\
\hline & PSNR & $\mathbf{r}$ & PSNR & $\mathbf{r}$ & PSNR & $\mathbf{r}$ & PSNR & $\mathbf{r}$ \\
\hline Noised & 20.18 & 0.8714 & 13.94 & 0.6266 & 11.64 & 0.4915 & 9.682 & 0.3583 \\
\hline Median & 27.22 & 0.9689 & 23.28 & 0.9242 & 21.09 & 0.8814 & 18.71 & 0.8127 \\
\hline Wiener & 26.95 & 0.9675 & 20.95 & 0.8751 & 18.58 & 0.7927 & 16.57 & 0.6828 \\
\hline WDF & 26.81 & 0.9657 & 23.57 & 0.9296 & 21.30 & 0.8874 & 18.82 & 0.8125 \\
\hline Bilateral & 25.12 & 0.9506 & 15.24 & 0.6796 & 12.31 & 0.5200 & 10.02 & 0.3719 \\
\hline BM3D & 31.40 & 0.9882 & 16.56 & 0.7349 & 12.91 & 0.5414 & 10.43 & 0.3824 \\
\hline DTCWT & 30.12 & 0.9841 & 25.51 & 0.9576 & 22.89 & 0.9345 & 20.13 & 0.8964 \\
\hline IFOA-DTCWT-BF & 27.70 & 0.9729 & 25.26 & 0.9574 & 22.87 & 0.9372 & 20.09 & 0.8973 \\
\hline
\end{tabular}

Table 4. Comparison of the five methods (Peppers with Gaussian noise).

\begin{tabular}{ccccccccccc}
\hline & \multicolumn{2}{c}{$\boldsymbol{\sigma}=\mathbf{0 . 0 1}$} & \multicolumn{2}{c}{$\boldsymbol{\sigma}=\mathbf{0 . 0 5}$} & \multicolumn{2}{c}{$\boldsymbol{\sigma = 0 . 1}$} & \multicolumn{2}{c}{$\boldsymbol{\sigma}=\mathbf{0 . 2}$} \\
\cline { 3 - 10 } Methods & Parameters & PSNR & $\mathbf{r}$ & PSNR & $\mathbf{r}$ & PSNR & $\mathbf{r}$ & PSNR & $\mathbf{r}$ \\
\hline Noised & 20.13 & 0.9039 & 13.75 & 0.6869 & 11.43 & 0.5489 & 9.576 & 0.4135 \\
Median & 28.29 & 0.9832 & 23.67 & 0.9522 & 21.18 & 0.9181 & 18.51 & 0.8607 \\
Wiener & 27.10 & 0.9780 & 20.85 & 0.9072 & 18.60 & 0.8406 & 16.71 & 0.7449 \\
WDF & 26.93 & 0.9770 & 23.57 & 0.9498 & 21.46 & 0.9182 & 19.12 & 0.8554 \\
Bilateral & 25.51 & 0.9686 & 15.05 & 0.7387 & 12.09 & 0.5792 & 9.921 & 0.4294 \\
BM3D & 31.67 & 0.9924 & 16.19 & 0.7812 & 12.61 & 0.5982 & 10.34 & 0.4420 \\
DTCWT & 30.51 & 0.9900 & 25.94 & 0.9729 & 23.55 & 0.9584 & 21.00 & 0.9369 \\
IFOA-DTCWT-BF & 28.12 & 0.9830 & 25.61 & 0.9722 & 23.49 & 0.9591 & 20.74 & 0.9279 \\
\hline
\end{tabular}

The image denoising results showed that IFOA-DTCWT-BF had powerful performance dealing with Gaussian noise. In Table 3, we could see that although DTCWT acquired good data of PSNR, the $r$ was not always better than the proposed method. Moreover, the proposed method got clearer edge information. Median filter, Wiener filter and WDF had general performance. BM3D was incapable of dealing with heavy noise. Bilateral filter was not good at denoising Gaussian noise and acquired worst results, while, in the proposed method, it played an important role, as shown in Figure 12. The edge information of denoised Lena images processed by DTCWT and the proposed method is shown in Figure 13. The denoised images are shown in Figures 14-16.

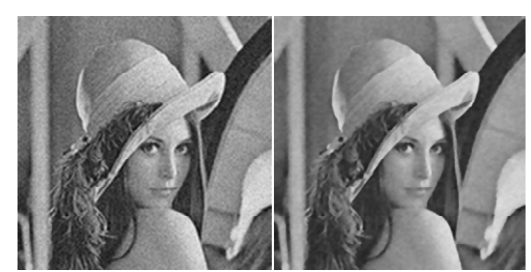

(A) $\sigma=0.01$

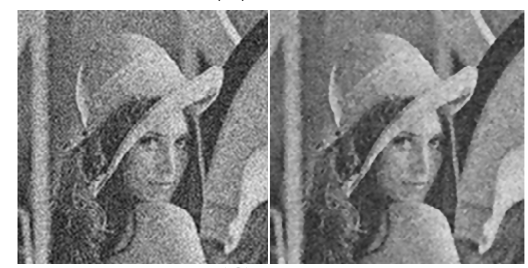

(C) $\sigma=0.1$

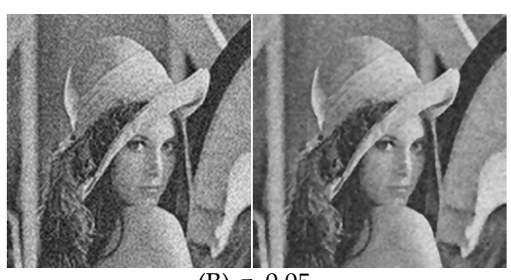

(B) $\sigma=0.05$

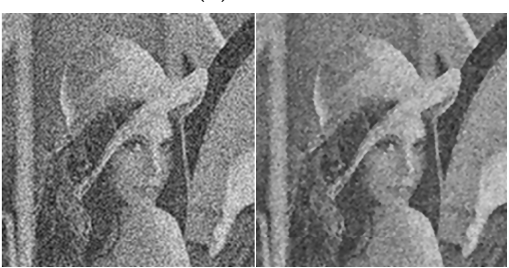

(D) $\sigma=0.2$

Figure 12. The effect of the bilateral filter: the left is no BF method and right is proposed method. (A) Lena of noise $\sigma=0.01$; (B) Lena of noise $\sigma=0.05$; (C) Lena of noise $\sigma=0.1$; (D) Lena of noise $\sigma=0.2$. 


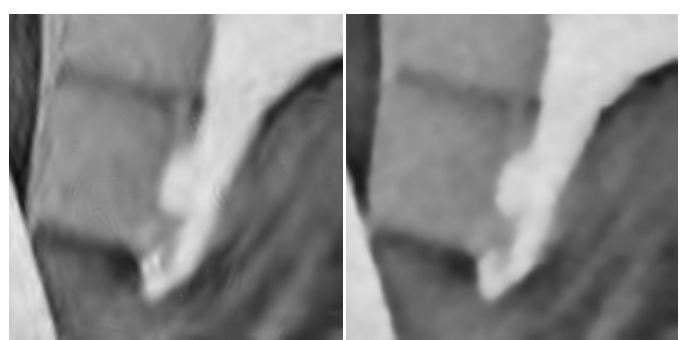

(A) $\sigma=0.01$
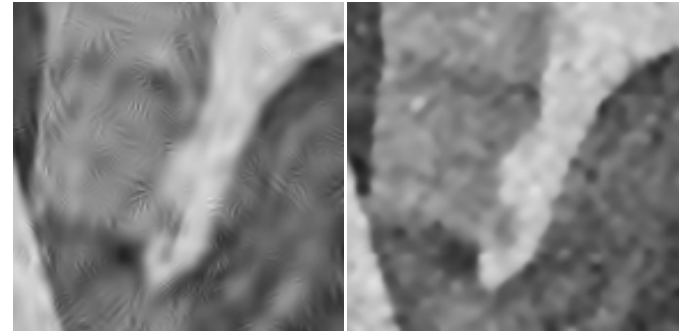

(C) $\sigma=0.1$

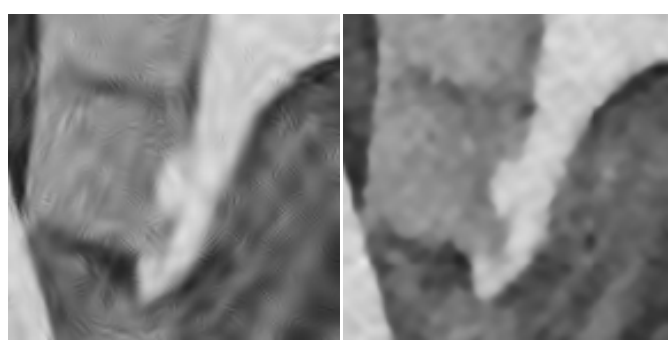

(B) $\sigma=0.05$

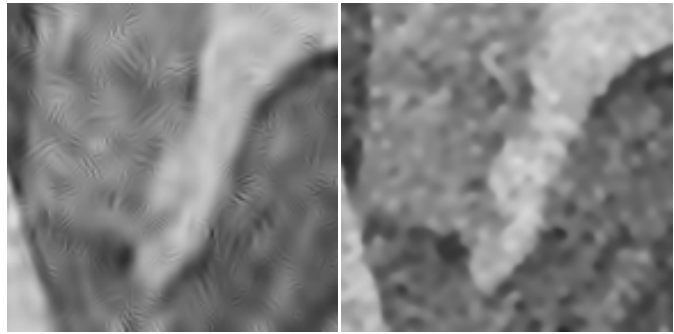

(D) $\sigma=0.2$

Figure 13. The edge information comparison of DTCWT and proposed method: left image is denoised by DTCWT, and right is proposed method. (A) Part of lena of noise $\sigma=0.01$; (B) Part of lena of noise $\sigma=0.05 ;(\mathbf{C})$ Part of lena of noise $\sigma=0.1 ;(\mathbf{D})$ Part of lena of noise $\sigma=0.2$.

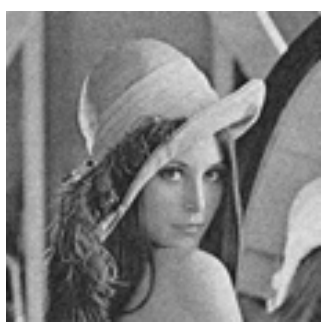

(A) $\sigma=0.01$

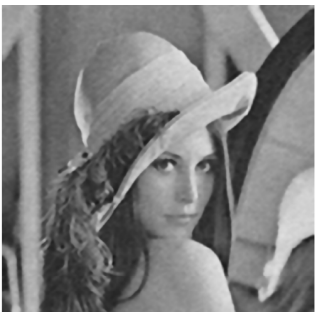

(E)Denoised, PSNR=28.66

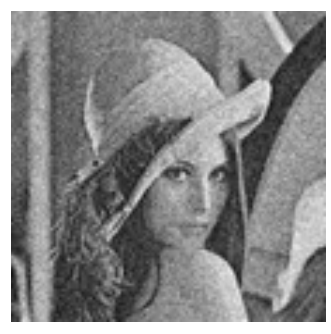

(B) $\sigma=0.05$

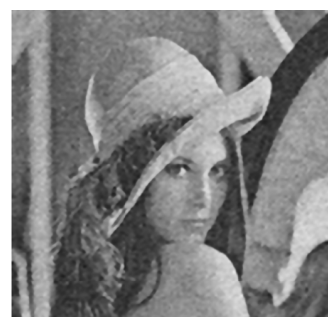

(F)Denoised, PSNR=26.34

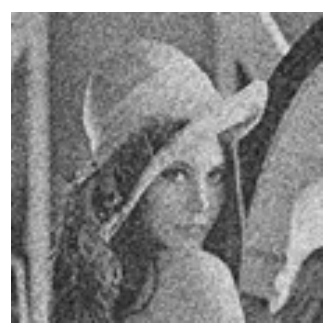

(C) $\sigma=0.1$

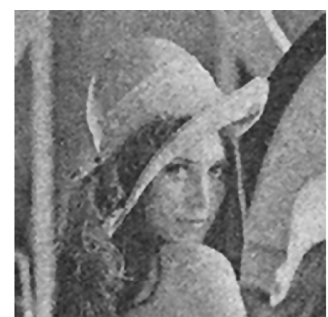

(G)Denoised, PSNR=24.15

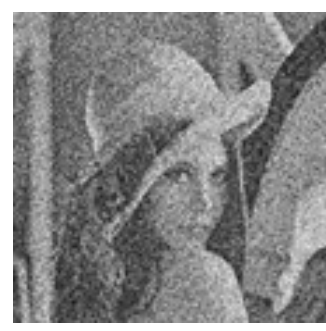

(D) $\sigma=0.2$

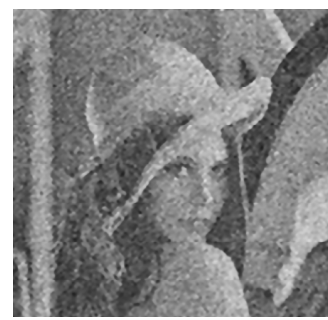

(H)Denoised, PSNR=21.57

Figure 14. Denosing results of Lena with Gaussian noise using IFOA-DTCWT-BF. (A) Lena of noise $\sigma=0.01$; (B) Lena of noise $\sigma=0.05$; (C) Lena of noise $\sigma=0.1$; (D) Lena of noise $\sigma=0.2$; (E) Denoised image of $\sigma=0.01 ;(\mathbf{F})$ Denoised image of $\sigma=0.05 ;(\mathbf{G})$ Denoised image of $\sigma=0.1 ;(\mathbf{H})$ Denoised image of $\sigma=0.2$. 


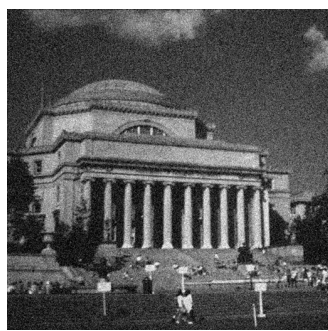

(A) $\sigma=0.01$

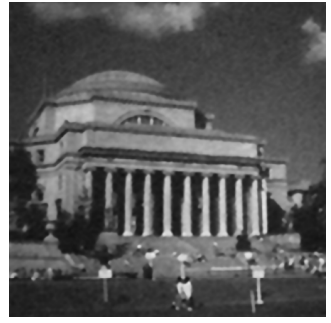

(E)Denoised, PSNR=27.70

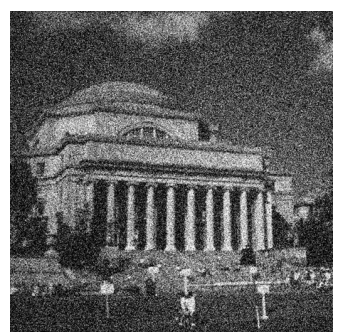

(B) $\sigma=0.05$

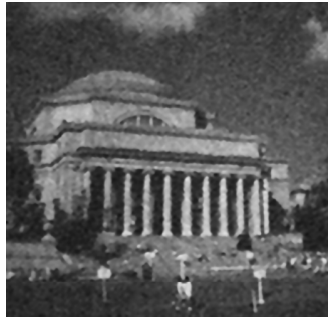

(F)Denoised, PSNR=25.26

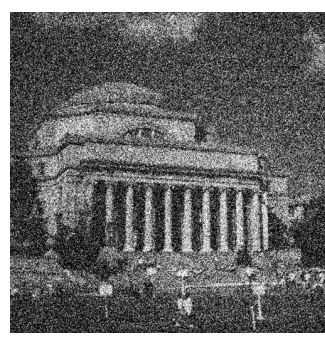

(C) $\sigma=0.1$

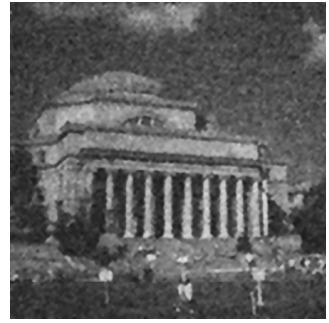

(G)Denoised, PSNR=22.87

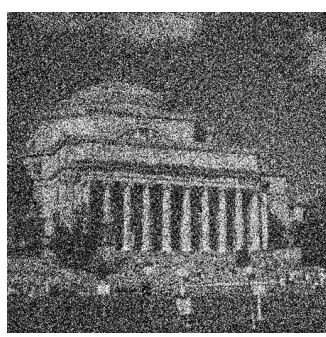

(D) $\sigma=0.2$

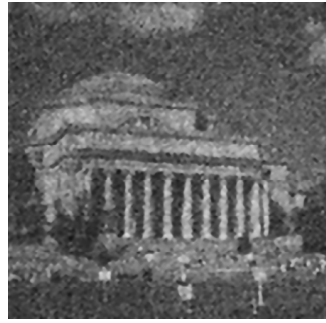

(H)Denoised, PSNR $=20.09$

Figure 15. Denosing results of Columbia with Gaussian noise using IFOA-DTCWT-BF. (A) Columbia of noise $\sigma=0.01$; (B) Columbia of noise $\sigma=0.05$; (C) Columbia of noise $\sigma=0.1$; (D) Columbia of noise $\sigma=0.2$; (E) Denoised image of $\sigma=0.01 ;(\mathbf{F})$ Denoised image of $\sigma=0.05 ;(\mathbf{G})$ Denoised image of $\sigma=0.1$;

(H) Denoised image of $\sigma=0.2$.

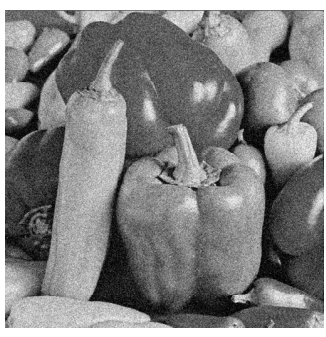

(A) $\sigma=0.01$

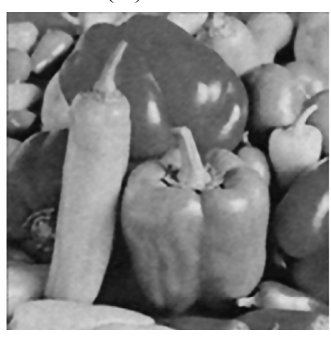

(E)Denoised, PSNR=28.12



(B) $\sigma=0.05$

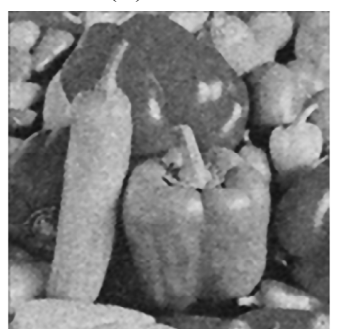

(F)Denoised, PSNR=25.61

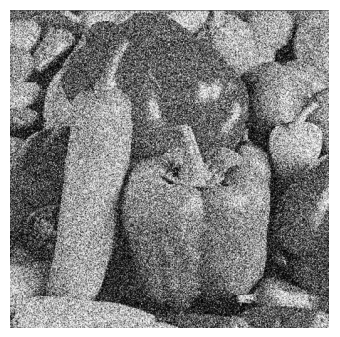

(C) $\sigma=0.1$

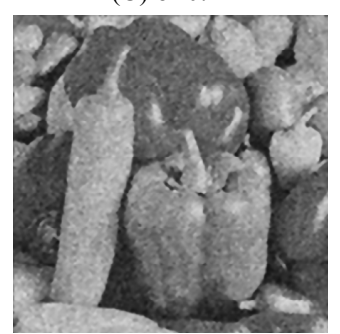

(G)Denoised, PSNR=23.49



(D) $\sigma=0.2$



(H)Denoised, PSNR=20.74

Figure 16. Denoising results of Peppers with Gaussian noise using IFOA-DTCWT-BF. (A) Peppers of noise $\sigma=0.01$; (B) Peppers of noise $\sigma=0.05$; (C) Peppers of noise $\sigma=0.1$; (D) Peppers of noise $\sigma=0.2$; (E) Denoised image of $\sigma=0.01 ;(\mathbf{F})$ Denoised image of $\sigma=0.05 ;(\mathbf{G})$ Denoised image of $\sigma=0.1$;

(H) Denoised image of $\sigma=0.2$.

\subsection{Experiment with Speckle Noise}

Speckle noise, which is known as multiplicative noise, added to original images forms noisy images. It can be described as: $g(x, y)=f(x, y) \cdot n(x, y)$, in which $n(x, y)$ is the multiplicative noise. The original images were polluted by Speckle noise using Matlab function IMNOISE in the experiment. Different noise variances were set to pollute the original image, and different methods were utilized to compare the denoising performances. Denoising results are listed in Tables 5-7. 
Table 5. Comparison of the six methods (Lena with Speckle noise).

\begin{tabular}{|c|c|c|c|c|c|c|c|c|}
\hline \multirow[b]{2}{*}{ Methods } & \multicolumn{2}{|c|}{$\sigma=0.01$} & \multicolumn{2}{|c|}{$\sigma=0.05$} & \multicolumn{2}{|c|}{$\sigma=0.1$} & \multicolumn{2}{|c|}{$\sigma=0.2$} \\
\hline & PSNR & $\mathbf{r}$ & PSNR & $\mathbf{r}$ & PSNR & $\mathbf{r}$ & PSNR & $\mathbf{r}$ \\
\hline Noised & 25.68 & 0.9637 & 18.83 & 0.8520 & 15.99 & 0.7526 & 13.25 & 0.6226 \\
\hline Median & 29.44 & 0.9839 & 25.82 & 0.9634 & 23.66 & 0.9410 & 21.27 & 0.9030 \\
\hline Wiener & 31.49 & 0.9899 & 24.92 & 0.9553 & 22.22 & 0.9180 & 19.60 & 0.8547 \\
\hline WDF & 28.76 & 0.9810 & 26.98 & 0.9713 & 25.40 & 0.9586 & 23.29 & 0.9328 \\
\hline Bilateral & 31.51 & 0.9900 & 22.05 & 0.9180 & 17.75 & 0.8100 & 14.19 & 0.6610 \\
\hline BM3D & 33.40 & 0.9935 & 26.79 & 0.9704 & 19.79 & 0.8683 & 15.08 & 0.6978 \\
\hline DTCWT & 31.88 & 0.9908 & 25.83 & 0.9633 & 23.65 & 0.9392 & 21.28 & 0.8954 \\
\hline IFOA-DTCWT-BF & 29.14 & 0.9829 & 28.41 & 0.9796 & 27.39 & 0.9749 & 25.55 & 0.9633 \\
\hline
\end{tabular}

Table 6. Comparison of the six methods (Columbia with Speckle noise).

\begin{tabular}{ccccccccccc}
\hline & \multicolumn{2}{c}{$\boldsymbol{\sigma}=\mathbf{0 . 0 1}$} & \multicolumn{2}{c}{$\boldsymbol{\sigma}=\mathbf{0 . 0 5}$} & \multicolumn{2}{c}{$\boldsymbol{\sigma = 0 . 1}$} & \multicolumn{2}{c}{$\boldsymbol{\sigma}=\mathbf{0 . 2}$} \\
\cline { 3 - 10 } Methods & Parameters & PSNR & $\mathbf{r}$ & PSNR & $\mathbf{r}$ & PSNR & $\mathbf{r}$ & PSNR & $\mathbf{r}$ \\
\hline Noised & 28.57 & 0.9782 & 21.68 & 0.9046 & 18.75 & 0.8313 & 15.93 & 0.7250 \\
Median & 29.27 & 0.9812 & 26.95 & 0.9678 & 25.35 & 0.9531 & 23.37 & 0.9260 \\
Wiener & 32.09 & 0.9899 & 26.16 & 0.9615 & 23.34 & 0.9283 & 20.72 & 0.8742 \\
WDF & 28.05 & 0.9743 & 27.22 & 0.9688 & 26.26 & 0.9610 & 24.93 & 0.9468 \\
Bilateral & 31.20 & 0.9879 & 25.01 & 0.9495 & 21.09 & 0.8861 & 17.47 & 0.7777 \\
BM3D & 33.08 & 0.9920 & 29.70 & 0.9825 & 24.13 & 0.9397 & 18.99 & 0.8292 \\
DTCWT & 31.55 & 0.9888 & 25.10 & 0.9517 & 22.40 & 0.9142 & 19.95 & 0.8554 \\
IFOA-DTCWT-BF & 28.21 & 0.9759 & 27.81 & 0.9735 & 27.29 & 0.9701 & 26.35 & 0.9630 \\
\hline
\end{tabular}

Table 7. Comparison of the six methods (Peppers with Speckle noise).

\begin{tabular}{ccccccccccc}
\hline & \multicolumn{2}{c}{$\boldsymbol{\sigma}=\mathbf{0 . 0 1}$} & \multicolumn{2}{c}{$\boldsymbol{\sigma}=\mathbf{0 . 0 5}$} & \multicolumn{2}{c}{$\boldsymbol{\sigma = 0 . 1}$} & \multicolumn{2}{c}{$\boldsymbol{\sigma}=\mathbf{0 . 2}$} \\
\cline { 3 - 10 } Methods & Parameters & PSNR & $\mathbf{r}$ & PSNR & $\mathbf{r}$ & PSNR & $\mathbf{r}$ & PSNR & $\mathbf{r}$ \\
\hline Noised & & & & & & & & \\
Median & 25.76 & 0.9715 & 18.89 & 0.8795 & 16.09 & 0.7965 & 13.42 & 0.6788 \\
Wiener & 30.14 & 0.9892 & 26.19 & 0.9678 & 23.81 & 0.9548 & 21.24 & 0.9212 \\
WDF & 31.51 & 0.9921 & 24.74 & 0.9632 & 22.03 & 0.9325 & 19.43 & 0.8792 \\
Bilateral & 28.04 & 0.9822 & 26.52 & 0.9748 & 25.22 & 0.9659 & 23.18 & 0.9459 \\
BM3D & 31.95 & 0.9928 & 22.01 & 0.9338 & 17.81 & 0.8464 & 14.34 & 0.7147 \\
DTCWT & 33.12 & 0.9945 & 26.20 & 0.9734 & 19.68 & 0.8918 & 15.28 & 0.7518 \\
IFOA-DTCWT-BF & 30.73 & 0.9905 & 24.40 & 0.9604 & 22.23 & 0.9351 & 20.13 & 0.8948 \\
\hline
\end{tabular}

As shown in Tables 5-7, when the noise variance of Speckle noise was 0.01, the DTCWT, BM3D, Wiener and bilateral filter did good jobs. However, the proposed method performed much better than other methods when removing the Speckle noise of big noise variances. The results indicated that the proposed method was insensitive to the Speckle noise. In other words, it can be adopted in any condition. All denoised images are shown in Figures 17-19. 


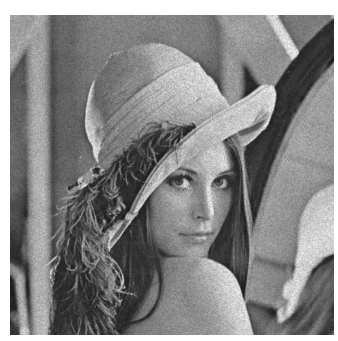

(A) $\sigma=0.01$

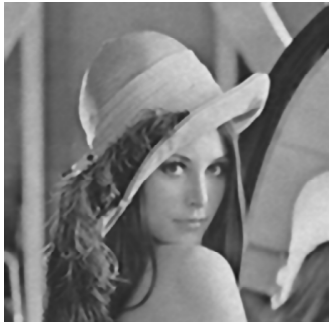

(E)Denoised, PSNR=29.14

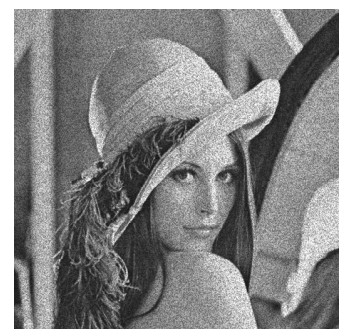

(B) $\sigma=0.05$

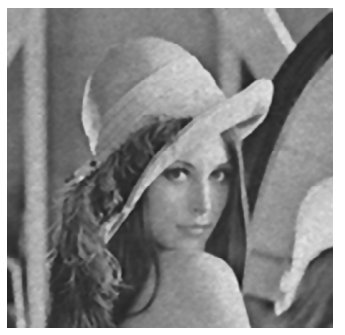

(F)Denoised, PSNR=28.41

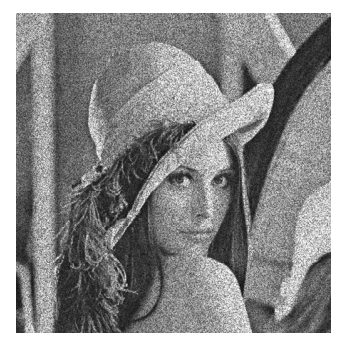

(C) $\sigma=0.1$

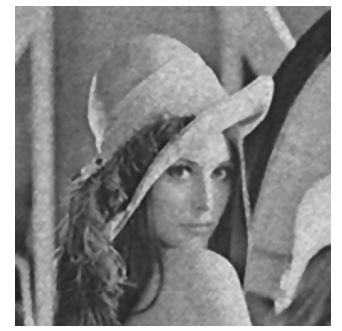

(G)Denoised, PSNR=27.39

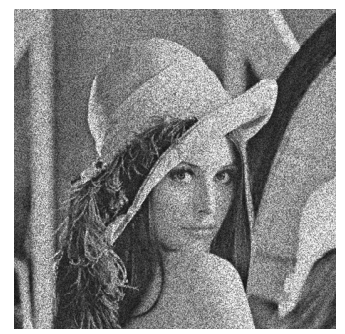

(D) $\sigma=0.2$

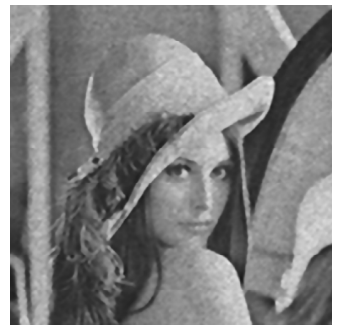

(H)Denoised, PSNR=25.55

Figure 17. Denoising results of Lena with Speckle noise using IFOA-DTCWT-BF. (A) Lena of noise $\sigma=0.01$; (B) Lena of noise $\sigma=0.05$; (C) Lena of noise $\sigma=0.1$; (D) Lena of noise $\sigma=0.2$; (E) Denoised image of $\sigma=0.01 ;(\mathbf{F})$ Denoised image of $\sigma=0.05 ;(\mathbf{G})$ Denoised image of $\sigma=0.1 ;(\mathbf{H})$ Denoised image of $\sigma=0.2$.

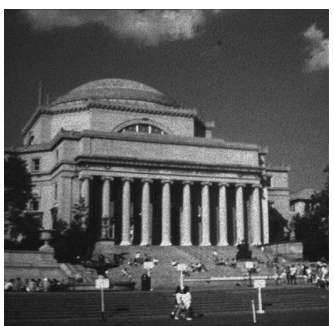

(A) $\sigma=0.01$



(E)Denoised, PSNR $=28.21$

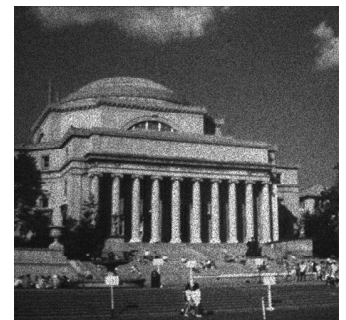

(B) $\sigma=0.05$

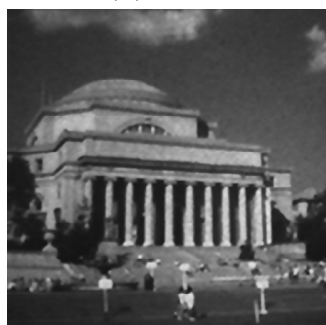

(F)Denoised, PSNR=27.81

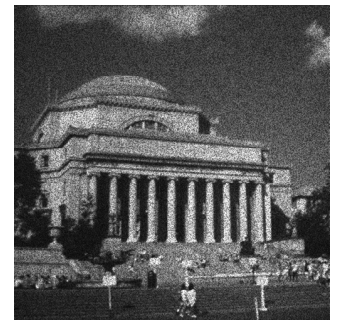

(C) $\sigma=0.1$

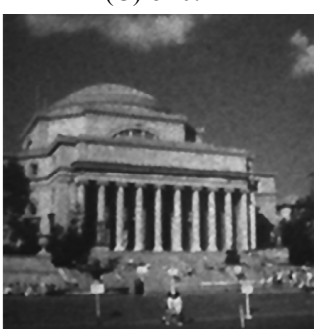

(G)Denoised, PSNR=27.29

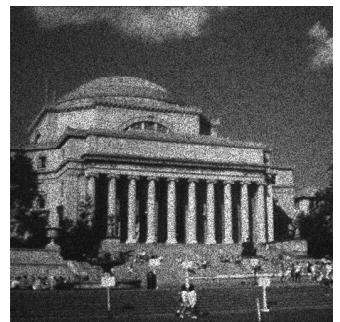

(D) $\sigma=0.2$

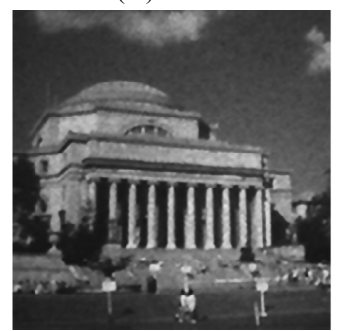

(H)Denoised，PSNR=26.35

Figure 18. Denoising results of Columbia with Speckle noise using IFOA-DTCWT-BF. (A) Columbia of noise $\sigma=0.01$; (B) Columbia of noise $\sigma=0.05$; (C) Columbia of noise $\sigma=0.1$; (D) Columbia of noise $\sigma=0.2$; (E) Denoised image of $\sigma=0.01 ;(\mathbf{F})$ Denoised image of $\sigma=0.05 ;(\mathbf{G})$ Denoised image of $\sigma=0.1$; (H) Denoised image of $\sigma=0.2$. 


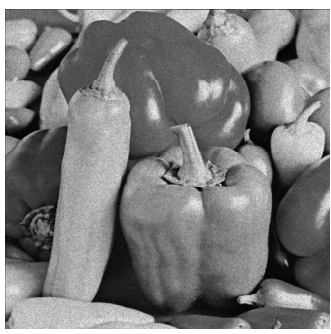

(A) $\sigma=0.01$

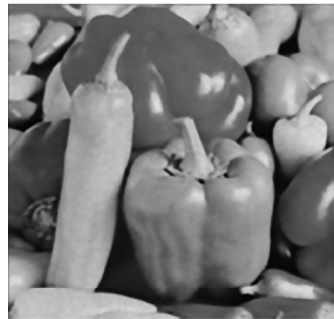

(E)Denoised, PSNR $=28.66$

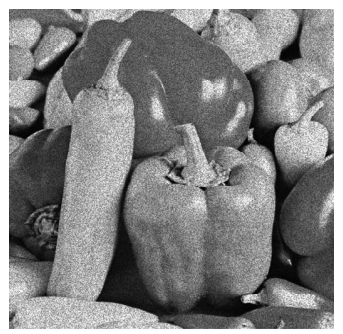

(B) $\sigma=0.05$



(F)Denoised, PSNR=28.01

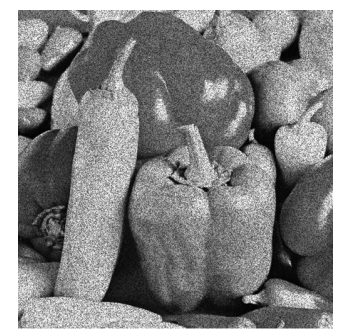

(C) $\sigma=0.1$

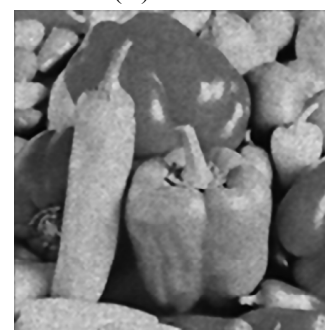

(G)Denoised, PSNR=27.14

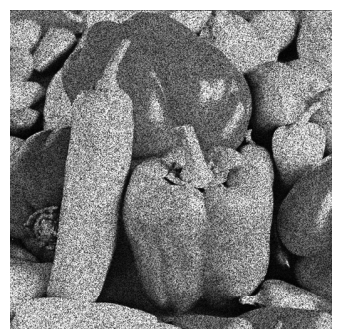

(D) $\sigma=0.2$

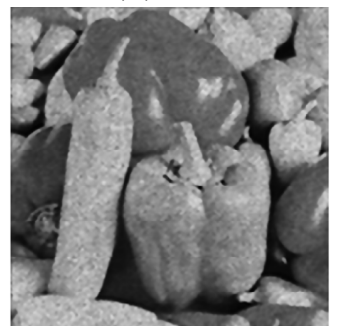

(H)Denoised, PSNR=25.45

Figure 19. Denoising results of Peppers with Speckle noise using IFOA-DTCWT-BF. (A) Peppers of noise $\sigma=0.01$; (B) Peppers of noise $\sigma=0.05$; (C) Peppers of noise $\sigma=0.1$; (D) Peppers of noise $\sigma=$ 0.2; (E) Denoised image of $\sigma=0.01$; (F) Denoised image of $\sigma=0.05$; (G) Denoised image of $\sigma=0.1$;

(H) Denoised image of $\sigma=0.2$.

\subsection{Application}

At present, the video monitoring of coal winning machine under the mine is getting more and more attention. However, using the camera for video monitoring is challenged by low illumination and coal dust environment in the mining face. When cutting the coal wall, the cutting unit of the mining machine produces great heat and radiates infrared ray. Thus, in 2016, the infrared thermal imager was applied to monitor the coal winning machine in Zhangjiakou Coal Mining Machinery Co., Ltd., Zhangjiakou, China. The infrared thermal imager taking into use in mining face could acquire clearer images than camera, as shown in Figure 20C, and it was more likely to realize the video monitoring and tracking of coal mining machine.



(A)Image from surface mining engineering

Figure 20. Cont. 


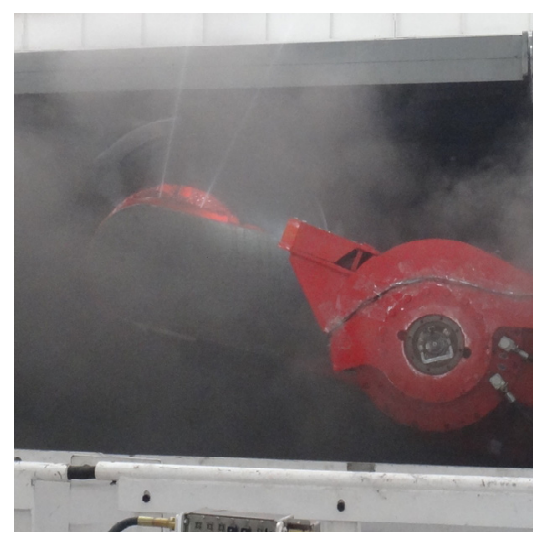

(B)Cutting unit of mining machine

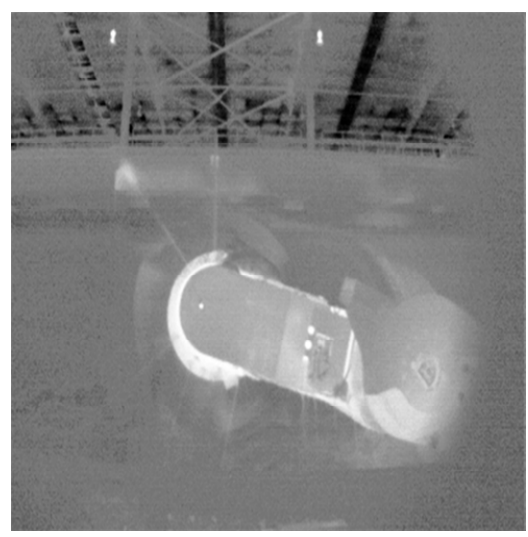

(C)Infrared thermal image of cutting unit

Figure 20. Original image form the surface experiment. (A) Image form surface mining engineering; (B) Cutting unit of mining machine; (C) Infrared thermal image of cutting unit.

However, the infrared thermal images have same unavoidable weaknesses. The low sharpness, noise problem and inhomogeneous are three major problems of the thermal images. Because of the low detectivity and spatial resolution of the thermal imaging system, the thermal images owns blurred visual effect. The random disturbances of the external environment like lamp light, dust, etc. cause the noise problem and the inconsistent of the response characteristics of each pixel in infrared detector results in the inhomogeneous of the thermal images. Before the infrared thermal image can be used for tracking coal mining machine, it needs to be denoised. In this work, the original infrared thermal images were denoised by the proposed method and other methods, and the denoised images are shown in Figure 21.

As the images were denoised, the processed images would have less information than before. Therefore, information entropy was used to represent the amount of information contained in the image: the greater the value of information entropy, the more information the image contains. As the noise in the images was wiped by denoising methods, the denoised images were smoother than before. The information entropy (Ie) and smoothness (Smo) were introduced to evaluate the images in Figure 21, which could be calculated as

$$
\begin{gathered}
I e=-\sum_{k=1}^{K} H_{k} \log H_{k} . \\
\text { Smo }=\frac{1}{M \times N} \sum_{i=1}^{m} \sum_{j=1}^{n} S_{b e s t}(i, j)
\end{gathered}
$$

where $K$ was the sum of gray scales in an image, $H_{k}$ is the proportion of the pixels of gray value $k$ in an image, and $S_{\text {best }}(i, j)$ was the average gray value of the pixels in the best active window.

The information entropy and smoothness of original images and processed images are plotted in Figure 22. As shown in Figure 22, we could find that the information entropies of images processed by different methods are almost the same. It showed the denoised images also had good quality and the major information of images was retained. In Figures 21 and 23, the images processed by proposed method had best smoothness. The smoothness of the images processed by IFOA-DTCWT-BF was 24.07, 24.28 and 23.41, which could not be acquired by other methods. It was beneficial for the following work of cutting unit tracking, as there were less unnecessary textures. The analysis results indicated that the images denoised by IFOA-DTCW-BF were better than other methods, which had better image textures and quality. 
(A)


WDF
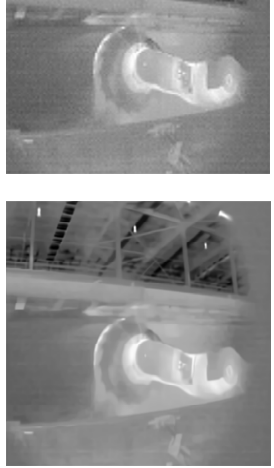

BM3D
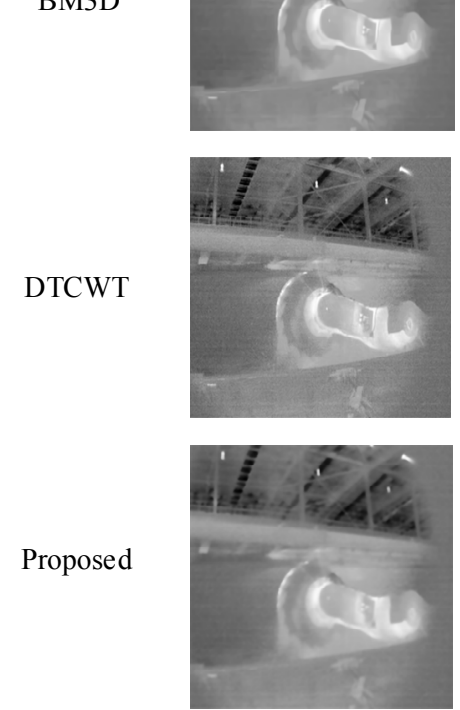

(B)
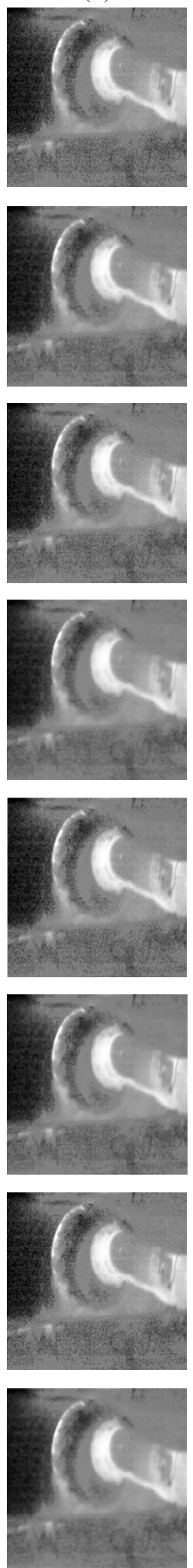

(C)
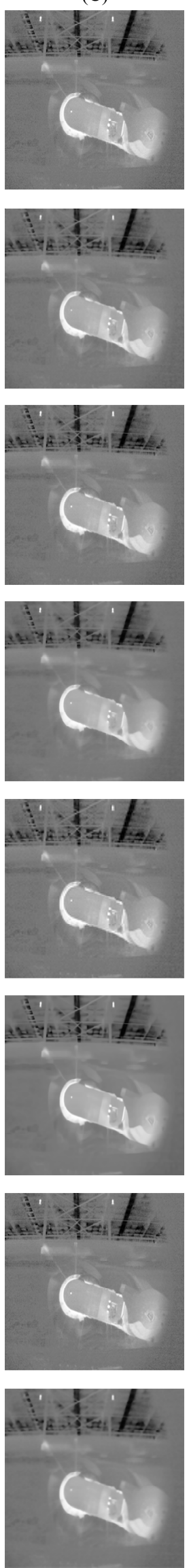

Figure 21. Original images and denoised images using different methods. (A) Infrared thermal image of cutting unit from the side; (B) Infrared thermal image of cutting unit from close distance; (C) Infrared thermal image of cutting unit from the front. 




Figure 22. Information entropy of images using different methods.

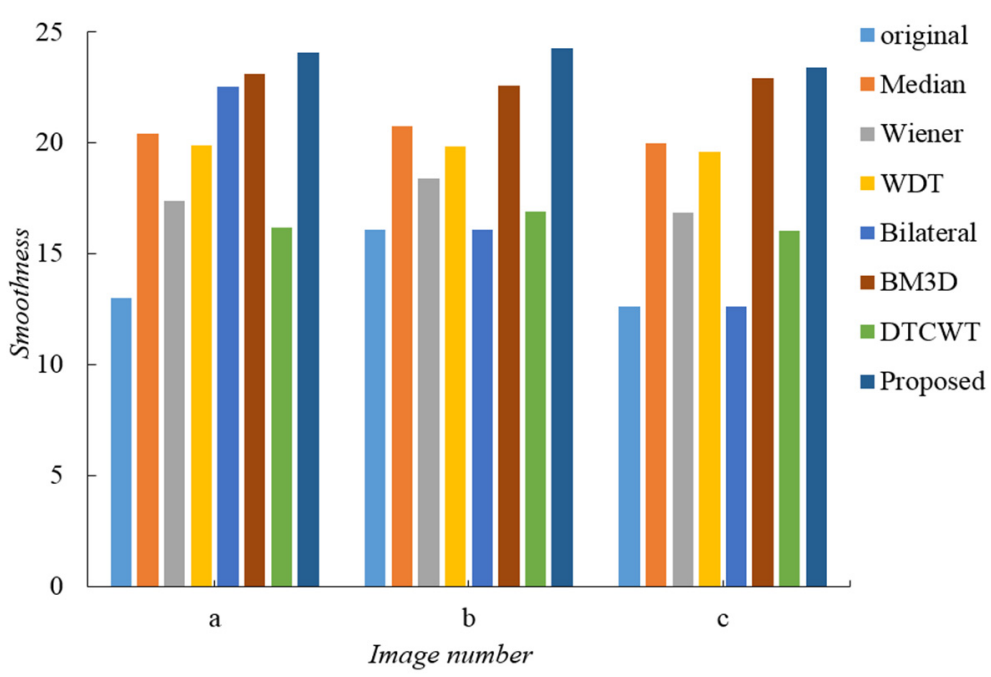

Figure 23. Smoothness of images using different methods.

\section{Conclusions and Future Work}

To eliminate noise from a noisy image without reference image and obtain good performance, this paper proposed a non-reference image denoising method based on enhanced dual-tree complex wavelet optimized by fruit fly algorithm and bilateral filter. In the absence of reference image, a hypothesis was proposed that the MSE of the denoising image and noisy image was supposed to increase as the noise is denoised. The IFOA searched the optimal threshold to gain best denoising performance. The bilateral filter was applied to process images in the late work, which can significantly improve the performance of images and enhance the edge information. To verify the performance of the proposed IFOA, it was compared with original FOA. Moreover, different inertia weights were tested to get the best inertia weight. As comparisons, six representative methods, DTCWT, BM3D, Median filter, Wiener filter, DWT and BF, were implemented. The results showed that the proposed denoising method outperformed other methods. Finally, the proposed IFOA-DTDWT-BF was applied to infrared thermal image of roller in running to test the actual effect.

This work is valuable for image processing, which provides a non-reference denoising method. However, there are also some shortcomings. On the one hand, the calculation duration is still a problem, although it is faster than other adaptive methods. On the other hand, the proposed algorithm adopts the MSE as the denoising evaluation index, which may have imperfections. To surmount these 
shortcomings, the authors intend to improve the method with more effective intelligence algorithms and form an evaluation function with several evaluation indexes to assess the quality of the denoised image. Besides, the authors plan to combine other efficient denoising methods with the proposed methods to create a better method and apply the method in the aspect of industrial vision in the future.

Acknowledgments: The authors would like to thank all of the reviewers for their constructive comments. The supports of National Natural Science Foundation of China (No. U1510117 and 51605477), National Key Basic Research Program of China (No. 2014CB046301), and the Priority Academic Program Development (PAPD) of Jiangsu Higher Education Institutions in carrying out this research are gratefully acknowledged.

Author Contributions: Zhongbin Wang and Yiwen Liu conceived and designed the experiments; Lin Zhang and Lei Si performed the experiments; Jing Xu and Chao Tan analyzed the data; and Yiwen Liu wrote the paper.

Conflicts of Interest: The authors declare no conflict of interest.

\section{References}

1. Fan, J.; Yi, H.; Xu, L.; Zhao, T. A Histogram-Based Denoising Algorithm in a Joint-Fourier Transform Correlator for Image Recognition. Photonic Optoelectron. 2012, 1-3. [CrossRef]

2. Walia, G.S.; Raza, S.; Gupta, A.; Asthana, R.; Singh, K. A novel approach of multi-stage tracking for precise localization of target in video sequences. Expert Syst. Appl. Int. J. 2017, 78, 208-224. [CrossRef]

3. Gan, S.; Wang, S.; Chen, Y.; Chen, X.; Xiang, K. Separation of simultaneous sources using a structural-oriented median filter in the flattened dimension. Comput. Geosci. 2016, 86, 46-54. [CrossRef]

4. Bhamre, T.; Zhang, T.; Singer, A. Denoising and covariance estimation of single particle cryo-em images. J. Struct. Biol. 2016, 195, 72-81. [CrossRef] [PubMed]

5. Dai, L.; Yuan, M.; Zhang, X. Speeding up the bilateral filter: A joint acceleration way. IEEE Trans. Image Process. 2016, 25, 2657-2672. [CrossRef] [PubMed]

6. Ran, Q.; Yuan, L.; Zhao, T. Image encryption based on nonseparable fractional fourier transform and chaotic map. Opt. Commun. 2015, 348, 43-49. [CrossRef]

7. Cui, S.S.; Chen, G. Terahertz digital holography image denoising using stationary wavelet transform. In Proceedings of the SPIE Conferences of the Photoelectronic Technology Committee of the Chinese Society of Astronautics, Changchun, China, 13-15 August 2014; Volume 9522.

8. Shensa, M.J. The discrete wavelet transform: Wedding the a trous and mallat algorithms. IEEE Trans. Signal Process. 1992, 40, 2464-2482. [CrossRef]

9. Kingsbury, N. The dual-tree complex wavelet transform: A new technique for shift invariance and directional filters. In Proceedings of the IEEE Digital Signal Processing Workshop, Bryce Canyon, UT, USA, 9-12 August 1998; pp. 2543-2560.

10. Kingsbury, N. Complex wavelets for shift invariant analysis and filtering of signals. Appl. Comput. Harmonic Anal. 2001, 10, 234-253. [CrossRef]

11. Shahdoosti, H.R.; Hazavei, S.M. Image denoising in dual contourlet domain using hidden markov tree models. Dig. Signal Process. 2017, 67, 17-29. [CrossRef]

12. Gong, W.G.; Liu, X.Y.; Li, W.H.; Li, J.F. Local adaptive image denoising based on double-density dual-tree complex wavelet transform. Opt. Precis. Eng. 2009, 17, 1171-1180. [CrossRef]

13. Liu, Y.; Jiang, M. Image denoising algorithm based on dtcwt and adaptive windows. In Proceedings of the 3rd IEEE International Conference on Computer Science and Information Technology, Chengdu, China, 9-11 July 2010; pp. 207-210.

14. Lee, S.H.; Lee, J. Optimization of three-dimensional wings in ground effect using multiobjective genetic algorithm. J. Aircr. 2011, 48, 1633-1645. [CrossRef]

15. Bououden, S.; Chadli, M.; Karimi, H.R. An ant colony optimization-based fuzzy predictive control approach for nonlinear processes. Inf. Sci. 2015, 299, 143-158. [CrossRef]

16. Wang, H.B.; Fan, C.C.; Tu, X.Y. Afsaocp: A novel artificial fish swarm optimization algorithm aided by ocean current power. Appl. Intell. 2016, 45, 1-16. [CrossRef]

17. Cheng, R.; Jin, Y. A social learning particle swarm optimization algorithm for scalable optimization. Inf. Sci. 2015, 291, 43-60. [CrossRef]

18. Li, M.D.; Zhao, H.; Weng, X.W.; Han, T. A novel nature-inspired algorithm for optimization. Adv. Eng. Softw. 2016, 92, 65-88. [CrossRef] 
19. Yang, X.S. Nature-Inspired Metaheuristic Algorithms, 2nd ed.; Luniver Press: Beckington, UK, 2010; pp. 92-116. ISBN 9781905986286.

20. Pan, W.T. A new fruit fly optimization algorithm: Taking the financial distress model as an example. Knowl. Based Syst. 2012, 26, 69-74. [CrossRef]

21. Lin, S.M. Analysis of service satisfaction in web auction logistics service using a combination of fruit fly optimization algorithm and general regression neural network. Neural Comput. Appl. 2013, 22, 783-791. [CrossRef]

22. Li, H.Z.; Guo, S.; Li, C.J.; Sun, J.Q. A hybrid annual power load forecasting model based on generalized regression neural network with fruit fly optimization algorithm. Knowl. Based Syst. 2013, 37, 378-387. [CrossRef]

23. Wang, L.; Shi, Y.; Liu, S. An improved fruit fly optimization algorithm and its application to joint replenishment problems. Expert Syst. Appl. 2015, 42, 4310-4323. [CrossRef]

24. Fan, F.; Ma, Y.; Li, C.; Mei, X.; Huang, J.; Ma, J. Hyperspectral image denoising with superpixel segmentation and low-rank representation. Inf. Sci. Int. J. 2017, 397-398, 48-68. [CrossRef]

25. Wu, Q.; Li, Y.; Lin, Y. The application of nonlocal total variation in image denoising for mobile transmission. Multimedia Tools Appl. 2017, 76, 1-13. [CrossRef]

26. Jia, Z.G.; Wei, M. A new TV-Stokes model for image deblurring and denoising with fast algorithms. J. Sci. Comput. 2017, 72, 1-20. [CrossRef]

27. Zhu, F.; Chen, G.; Hao, J.; Heng, P.A. Blind image denoising via dependent dirichlet process tree. IEEE Trans. Pattern Anal. Mach. Intell. 2017, 39, 1518-1531. [CrossRef] [PubMed]

28. Papageorgiou, G.; Bouboulis, P.; Theodoridis, S. Robust non-linear regression: A greedy approach employing kernels with application to image denoising. IEEE Trans. Signal Process. 2016, 99. [CrossRef]

29. Zhang, L.; Zhou, X.; Wang, Z.; Tan, C.; Liu, X. A nonmodel dual-tree wavelet thresholding for image denoising through noise variance optimization based on improved chaotic drosophila algorithm. Int. J. Pattern Recognit. Artif. Intell. 2017, 31. [CrossRef]

30. Jung, C.; Yang, Q.; Sun, T.; Fu, Q.; Song, H. Low light image enhancement with dual-tree complex wavelet transform. J. Vis. Commun. Image Represent. 2016, 42. [CrossRef]

31. Lama, R.K.; Choi, M.R.; Kwon, G.R. Image interpolation for high-resolution display based on the complex dual-tree wavelet transform and hidden markov model. Multimedia Tools Appl. 2016, 75, 1-12. [CrossRef]

32. Zhang, X. Image denoising using dual-tree complex wavelet transform and wiener filter with modified thresholding. J. Sci. Ind. Res. 2016, 75, 687-690.

33. Wei, T.; Gao, Q.; Ma, N.; Li, N.; Wang, J.; Lei, P.; Ji, X. Feature-level image fusion through consistent region segmentation and dual-tree complex wavelet transform. J. Imaging Sci. Technol. 2016. [CrossRef]

34. Zhao, Z.; Dong, J.; Li, H. A novel biometric image encryption algorithm based on compressed sensing and dual-tree complex wavelet transform. In Proceedings of the Eighth International Conference on Digital Image Processing, Chengdu, China, 20-22 May 2016.

35. Tian, Y.; Luo, J.; Zhang, W.; Jia, T.; Wang, A.; Li, L. Multifocus image fusion in q-shift dtcwt domain using various fusion rules. Math. Probl. Eng. 2016, 2016, 1-12. [CrossRef]

36. Meng, T.; Pan, Q.K. An improved fruit fly optimization algorithm for solving the multidimensional knapsack problem. Appl. Soft Comput. 2017, 50, 79-93. [CrossRef]

37. $\mathrm{Xu}, \mathrm{C}$. An improved fruit fly optimization algorithm and its application of pid parameters tuning. J. Inf. Comput. Sci. 2015, 12, 3647-3654. [CrossRef]

38. Yang, Y.; Luo, Z.; Xie, C. Study and application on fruit fly optimization algorithm optimized general regression neural network in mined-out area stability analysis. World Sci. Tech. R D 2015, 3, 230-234. [CrossRef]

39. Wu, L.; Cao, G. Seasonal svr with foa algorithm for single-step and multi-step ahead forecasting in monthly inbound tourist flow. Knowl. Based Syst. 2016, 110, 157-166. [CrossRef]

40. Niu, J.; Zhong, W.; Liang, Y.; Luo, N.; Qian, F. Fruit fly optimization algorithm based on differential evolution and its application on gasification process operation optimization. Knowl. Based Syst. 2015, 88, 253-263. [CrossRef]

41. Niu, D.; Ma, T.; Liu, B. Power load forecasting by wavelet least squares support vector machine with improved fruit fly optimization algorithm. J. Comb. Optim. 2016, 33, 1-22. [CrossRef] 
42. Xu, J.; Wang, Z.; Tan, C.; Si, L.; Liu, X. A novel denoising method for an acoustic-based system through empirical mode decomposition and an improved fruit fly optimization algorithm. Appl. Sci. 2017, 7, 215. [CrossRef]

43. Zhang, Y.; Cui, G.; Wu, J.; Pan, W.T.; He, Q. A novel multi-scale cooperative mutation fruit fly optimization algorithm. Knowl. Based Syst. 2016, 114, 24-35. [CrossRef]

44. Wang, L.; Liu, R.; Liu, S. An effective and efficient fruit fly optimization algorithm with level probability policy and its applications. Knowl. Based Syst. 2016, 97, 158-174. [CrossRef]

45. Şendur, L.; Selesnick, I.W. Bivariate shrinkage functions for wavelet-based denoising exploiting interscale dependency. IEEE Trans. Signal Process. 2002, 50, 2744-2756. [CrossRef]

(C) 2017 by the authors. Licensee MDPI, Basel, Switzerland. This article is an open access article distributed under the terms and conditions of the Creative Commons Attribution (CC BY) license (http://creativecommons.org/licenses/by/4.0/). 